\title{
Exosomal Long Noncoding Rna AGAP2-AS1 Regulates Trastuzumab Resistance via Inducing Autophagy in Breast Cancer
}

\section{Mingli Han}

Zhengzhou University

Hongbo Qu

Wuxi People's Hospital of Chenzhou City

Hui Cao

Zhengzhou University

Xiangke Li

Zhengzhou University

Dongwei Dou

Zhengzhou University

Xue Yang

Zhengzhou University

Yunqing Yang

Zhengzhou University

$\mathrm{Na}$ Han

Zhengzhou University

Mulin Ye

Hainan General Hospital

Jing Han

Hainan General Hospital

Fan Zhang

Hainan General Hospital

Wei Wang

Hainan General Hospital

Yichao Ding

Hainan General Hospital

Mingwei Xie

Hainan General Hospital

\section{Xueke Qian}

Zhengzhou University

Huaying Dong ( $\nabla$ dr_dhy@163.com ) 


\section{Research}

Keywords: breast cancer, trastuzumab resistance, AGAP2-AS1, ATG10, ELAVL1

Posted Date: June 12th, 2020

DOI: https://doi.org/10.21203/rs.3.rs-34252/v1

License: (c) (1) This work is licensed under a Creative Commons Attribution 4.0 International License. Read Full License 


\section{Abstract}

Background: Trastuzumab has been widely used for treatment of HER-2-positive breast cancer patients, however, the clinical response has been restricted due to emergence of resistance. Recent studies indicate that long noncoding RNA AGAP2-AS1 (IncRNA AGAP2-AS1) plays an important role in cancer resistance. However, the precise regulatory function and therapeutic potential of AGAP2-AS1 in trastuzumab resistance is still not defined.

Methods: Trastuzumab resistant cells were established. RNA sequencing and qRT-PCR were performed to identify the target gene of AGAP2-AS1. Mass spectrometry, RNA pulldown and RNA immunoprecipitation assays were performed to verify the direct interactions among AGAP2-AS1 and other associated targets, such as embryonic lethal abnormal version like RNA binding protein 1 (ELAVL1) and autophagy related 10 (ATG10). In vitro and in vivo experimental assays were done to clarify the functional role of exosomal AGAP2-AS1 in trastuzumab resistance.

Results: AGAP2-AS1 promotes and disseminates trastuzumab resistance via packaging into exosomes. Exosomal AGAP2-AS1 induces trastuzumab resistance via modulating ATG10 expression and autophagy activity. Mechanically, AGAP2-AS1 is associated with ELAVL1 protein. The AGAP2-AS1-ELAVL1 complex could directly bind to the promoter region of ATG10, inducing H3K27ac and H3K4me3 enrichment, which finally activates ATG10 transcription. AGAP2-AS1-targeting antisenseoligonucleotides (ASO) substantially increased trastuzumab-induced cytotoxicity. Clinically, increased expression of serum exosomal AGAP2-AS1 was associate with poor response to trastuzumab treatment.

Conclusion: ExosomalAGAP2-AS1 increased trastuzumab resistance via promoting ATG10 expression and inducing autophagy. Therefore, AGAP2-AS1 may serve aspredictive biomarker and therapeutic target for HER-2+ breast cancer patients.

\section{Background}

Human epidermal growth factor receptor 2 (HER-2) positive breast cancer (BC) is one of the most common types of BC, and HER-2 is amplified or overexpressed in 15 to $20 \%$ of all BC patients [1]. Currently, the use of adjuvant trastuzumab is the standard of care for these patients [2]. However, a very limited part of patients responds well to trastuzumab and over $60 \%$ become resistance[3]. Thus, finding novel therapeutic approaches and new targets to promote trastuzumab cytotoxicity are urgently needed.

Long noncoding RNAs (IncRNAs) are now described to have many functions and involved in tumor progression and response to therapy [4]. Evidence is mounting that numerous IncRNAs are involved in regulating trastuzumab resistance, such as GAS5, UCA1 and ATB[5-8]. Previously, we demonstrated the essential role of AFAP1-AS1 in trastuzumab resistance. AFAP1-AS1 could interact with AUF1, and then activate ERBB2 translation [9]. RNA-binding proteins (RBPs) are playing important functions in cancersby regulating the messenger RNA (mRNA) stability and splicing[10]. RBPsregulate the step of RNA processing to modulate the progression ofBC [11]. For instance, RNA-binding protein CUGBP1 controls the 
differential INSR splicing in molecular subtypes of BC cells and affects cell aggressiveness[12]. And RNAbinding protein RBM38 increases PTEN expression via enhancing its mRNA stability in BC[13].Those studies along with ours indicate that IncRNAs are critical regulators for trastuzumab resistanceby binding with RBPs.

Autophagy is a well-known cellular process which is accompanied by various pathologic processes and biological reactions, including hypoxia, cancer, nutritional deficiencies and other cellular stresses[14]. Thus, it is easily understood that autophagy plays important roles during drug treatment and resistance. Learning how autophagy participates in trastuzumab resistance could provide new insights into overcoming resistance and developing new therapeutic targets[15]. By modulating key autophagy-related proteins expression, IncRNAs plays essential roles in regulating autophagy [16]. Therefore, finding the regulatory relationships between IncRNAs and autophagy in trastuzumab resistance may provide new thoughts in BC treatment.

In our previous study, we revealed a critical role of AGAP2-AS1 in trastuzumab resistance [17]. Here, we continueto explore the underlyingregulatory mechanism of AGAP2-AS1 in BC trastuzumab resistance through binding with RBPs and regulation of autophagy, which may help us obtain new insights into the clinical potential of AGAP2-AS1 in BC therapy.

\section{Materials And Methods}

\section{Patient samples}

Overall, a cohort of serum samples obtained from 90 HER-2 positive patients were included from March 2012 to January 2015, to determine the expression of serum exosomal AGAP2-AS1 for trastuzumab response. Patients include 45 responding and 45 non-responding patients according to iRECIST criteria [18]. Written-informed consent was obtained from all patients and the study protocol was approved by theResearch Scientific Ethics Committee of The First Affiliated Hospital of Zhengzhou University,The First People's Hospital of Chenzhou City and Hainan General Hospital.

\section{Cell culture and treatment}

The human HER-2 positive BC cell lines SKBR-3 and BT474 were obtained from the Cell Bank of the Chinese Academy of Sciences (Shanghai, China). Cells were cultured in RPMI-1640 medium (Gibco, CA, USA) supplemented with $10 \%$ fetal bovine serum (Australia Origin, Gibco, Carlsbad, CA, USA) and 1\% penicillin/streptomycin (Solarbio, Beijing, China). The cells were grown in a humidified atmosphere of $5 \%$ $\mathrm{CO}_{2}$ and $95 \%$ air at $37^{\circ} \mathrm{C}$. Trastuzumab (Herceptin) was purchased from Roche (Basel, Switzerland) and used by dissolving in phosphate-buffered saline (PBS). The trastuzumab-resistant cell lines, SKBR-3-TR and BT474-TR, originated from SKBR-3 and BT474 cells, respectively, were established as previously described [19]. 
Total RNA was isolated from the cultured cell lines or clinical samples by use of Trizolreagent (Invitrogen, Carlsbad, CA), and subjected to reverse transcription as requested(Takara, Shiga, Japan). The RNA concentration and purity were measured by Nanodrop 2000 (Thermo Scientific, Waltham, MA, USA). The

expression levels of mRNA and IncRNA were detected by the PrimeScript ${ }^{\mathrm{TM}}{ }_{\mathrm{RT}}$ reagent kit (Takara, Dalian, China). qRT-PCR was carried out using the SYBR Premix Ex Taq ${ }^{\mathrm{TM}} \mathbb{\nabla}$ (Takara, Dalian, China) andstandardized using GAPDH as reference gene.All reactions were carried out in triplicate. The relative expression was calculated using the $2^{-\triangle \Delta \mathrm{ct}}$ method. PCR primers were listed in Additional file 1: Table S1.

\section{Plasmid construction and cell transfection}

For knockdown ofAGAP2-AS1 and ATG10, small interfering RNA (siRNA) was purchased from GenePharma (Shanghai, China). To construct a plasmid overexpressing AGAP2-AS1, the full-length human AGAP2-AS1 sequence was synthesized and subcloned into the pEX-3 vector (GenePharma, Shanghai, China). ThepENTER-ATG10 vector used for overexpression ofATG10 mRNA was provided by Vigene BioSciences, followed by transfection into BC cells using Lipofectamine 2000 for $48 \mathrm{~h}$ (Invitrogen). For stably overexpressing or silencing AGAP2-AS1, we constructed lentivirus-based vectors from Genechem (Shanghai, China). The sequences of oligonucleotides are presented in Additional file 1: Table S1.

\section{CCK8 assay}

The cells transfected accordingly were seeded in a 96-well plate, the cells were cultured for $72 \mathrm{~h}$ with different dose of trastuzumab. After that, $10 \mathrm{mg} / \mathrm{mL}$ of CCK8 reagent (Dojindo, Kumamoto, Japan) was supplemented for cell viability detection according to manufacturer's protocols. The absorbance at 450 $\mathrm{nm}$ was measured with a microplate reader. The dose for the half survival was represented the halfmaximal inhibitory concentration (IC50) of trastuzumab in SKBR-3-TR and BT474-TR cells.

\section{Isolation and identification of exosomes}

Cell supernatant was collected andsequentially centrifuged under $3000 \mathrm{~g}$ for $15 \mathrm{~min}$ at $4^{\circ} \mathrm{C}$ after taken out from $-80^{\circ} \mathrm{C}$ and filtered through a $0.45-\mu \mathrm{m}$ pore polyvinylidene fluoride filter (Millipore, Billerica, Mass). Then, $63 \mu$ I ExoQuick ${ }^{\mathrm{TM}}$ exosome precipitation solution (SBI, USA) was added into $250 \mu \mathrm{l}$ serum followed by incubation at $4^{\circ} \mathrm{C}$ for $50 \mathrm{~min}$. After centrifugation at $1500 \mathrm{~g}$ for 30 minutes, exosomes were collected. $50 \mu$ phosphate-buffered saline (PBS) was used to resuspend the exosome pellets obtained from the former step. Exosome isolated from serum of BC patients were done with similar procedures.

The sample of exosomes was identified through transmission electron microscopy, nanoparticle tracking analysis (NTA, Malvern Panalytical). Exosomes were loaded on a Formvar-carbon-coated electron microscope grid(Polysciences) for $30 \mathrm{~min}$. Then the grid was washed in PBS and fixed in $2 \%$ glutaraldehyde(Sigma Aldrich) for $10 \mathrm{~min}$. The grid was subsequently washed in PBS for 5 times andcounter-stained with $2 \%$ uranyl acetate (Sigma Aldrich) for $1 \mathrm{~min}$. Air-dried grids wereviewed with a Hitachi transmission electron microscope. 


\section{RNA sequencing analysis}

Total RNA was extracted from wild type or AGAP2-AS1-knock out SKBR-3 cells using RNeasy mini kit (Qiagen, Germany). The paired-end libraries were constructed by using TruSeq ${ }^{\text {TM }}$ RNA Sample Preparation Kit (Illumina, USA) according to the manufacture'ssample preparation guide. The mRNA expression profiles of the treated cells were determined using the Illumina NovaSeq 6000 (Illumina, USA) following the manufacturer's instructions. The library construction and sequencing were performed at Shanghai Sinomics Corporation (Shanghai, China).Cuffdiff was used to evaluate differentially expressed genes. The differentially expressed genes were selected using the following filter criteria: $P \leq 0.05$ and fold change $\geq 2$.

\section{Subcutaneous tumorigenicity assay}

4-week-old female BALB/c nude mice were purchased from the Beijing Vital River Laboratory Animal Technology and housed in specific pathogen-free barrier facilities. Fifteen mice were randomly assigned to two exosome-treated groups and one control group ( 5 mice/group). Mice were subcutaneous injected SKBR-3-TR cells $\left(1 \times 10^{6}\right.$ cells in $0.1 \mathrm{ml}$ physiological saline) in the flank. Each group received PBS, SKBR-3TR-EXO ${ }_{\text {Vector }}$ or SKBR-3-TR-EXO AGAP2-AS1 $_{1}$ treatmentsevery 3 days for 5 weeks. Meanwhile, each group received intraperitoneal treatment of trastuzumab once every two days for 4 weeks. After 4 weeks postinjection, mice were imaged using a luminescence imaging system. At the end of imaging, all mice were euthanized by carbon dioxide inhalation, followed by cervical dislocation to ensure death. Then, tumors were surgically dissected and weighted. Tumor volume $\left(\mathrm{mm}^{3}\right)=0.5 \times$ width $^{2} \times$ length .

To test the therapeutic role of AGAP2-AS1, ASO was used for treatment of xenograft tumors through tail vein injection. Briefly, $5 \times 10^{5}$ SKBR-3-TR cells in $100 \mu \mathrm{L}$ of sterile PBS were injected directly into the mammary fat pads of mice to establish in situ xenograft. Then, mice were divided into 3 groups $(n=5$ in each group) after the xenograft was established. ASO was used at the concentration of $10 \mathrm{nmol}$, twice a week.

All experimental procedures were conducted in conformity with the ethical standards of national and international guidelines and policies. This study was approved by the Institute Animal Care and Use Committee of The First Affiliated Hospital of Zhengzhou University (Zhengzhou, China).

\section{RNA pulldown}

AGAP2-AS1 was transcribed using T7 RNA polymerase in vitro (Ambio Life) followed by the purification with RNeasy Plus Mini Kit (Qiagen) treatment with DNase I (Qiagen). The cell lysates were freshly prepared using Magnetic RNA-Protein Pull-Down Kit (Thermo Fisher Scientific, Cat\# 20164). Cell protein extracts were collected and mixed with the biotinylated

RNA probes for AGAP2-AS1 in magnetic beads. Resultswere analyzed by sliver staining and mass spectrometry (ekspertTMnanoLC; AB Sciex TripleTOF 5600-plus; SCIEX). Data were analyzed using 
Proteinpilot software (https://omictools.com/proteinpilot-tool). The retrieved protein ELAVL1 was further validated by standard western blot.

\section{RNA immunoprecipitation (RIP) and Chromatin immunoprecipitation (ChIP)}

Using EZ-Magna RIP RNA Binding Protein Immunoprecipitation Kit,RIP assay was implemented as instructed by supplier (Millipore, Billerica,MA). Cell lysates were reaped for incubation with the ELAVL1 (cat. no. ab200342, Abcam, Cambridge, MA) antibody in magnetic beads, using IgG antibody as negativecontrol. All precipitates wereexamined by qRT-PCR.

For ChIP assay, the Millipore EZ ChIP Kit (Millipre) was used according to the manufacture's guideline. After fixation with 4\%formaldehyde, cells were lysed by using RIPA buffer (Beyotime, Shanghai, China). Then, DNA fragments (length of 200-1000 bp) were obtained by treatment with ultrasonic followed byimmunoprecipitation usinganti-H3K27ac antibody (Abcam, cat. no. ab4729), H3K4me3 antibody (Abcam, cat. no. ab8580) and the negative control IgG antibody (EMD Millipore, cat. no. 12-371) overnight at $4^{\circ} \mathrm{C}$. The cross-linked chromatin was then acquired and detected via qRT-PCR after route elution. Experiments were performed in triplicate.

\section{RNA fluorescent in situ hybridization (RNA-FISH)}

The colocalization of AGAP2-AS1 and ELAVL1 were confirmed by fluorescence staining. Briefly, SKBR-3 cells were seeded on a glass-bottomed confocal plate and cultured overnight. After fixation with 4\% PFA and permeabilization with $0.5 \%$ Triton, hybridization was carried out overnight with the AGAP2-AS1 probes conjugated with Alexa Fluor 555 (Invitrogen, CA,USA) at $37^{\circ} \mathrm{C}$ in $2 \times S S C, 10 \%$ formamide and $10 \%$ dextran. Subsequently, Anti-ELAVL1 was incubated in the dark overnight followed by incubation with secondary antibody for $1 \mathrm{~h}$. Finally, the nuclei were stained by DAPI and the images were captured under a Nikon A1Si Laser Scanning Confocal Microscope (Nikon Instruments Inc, Japan).

\section{Immunohistochemistry (IHC) analysis}

For IHC, the formalin-fixed, paraffin-embedded sections were dewaxed and rehydrated aspreviously described [17], and then treated with $3 \%$ hydrogen peroxide followed by EDTA buffer for antigenretrieval. The sections were then blocked in goat serum for $30 \mathrm{~min}$, incubated with rabbit anti ATG10 antibody (Abcam, cat. no. ab229728) at $4^{\circ} \mathrm{C}$ overnight and subsequent with horseradish peroxidaseconjugatedsecondary antibodies for $30 \mathrm{~min}$ at room temperature. Finally, the sections were stained withthe DAB substrate and hematoxylin. Images were recorded by Nikon Eclipse Ti microscope.

\section{Western Blot analysis}

Protein extraction were performed using RIPA lysis buffer (Pierce, IL, USA) containingprotease inhibitor (Roche, CA, USA). Protein extracts were subjected to $10 \%$ SDS-polyacrylamide gel electrophoresis followed by electro-transfer to polyvinylidenedifluoride membrane. After $1 \mathrm{~h}$ of pre-membrane blocking with $5 \%$ BSA, the proteins wereincubated with respective primary antibodies at $4^{\circ} \mathrm{C}$ overnight followed by 
secondaryantibodies incubation at room temperature for $1 \mathrm{~h}$. The detection of proteins was carried outusing ECL reagent.

\section{Statistical analysis}

Each experiment contained at least 3 individual bio-replications. Datawere all given as the mean \pm standard deviation (SD) and processed withPrism Version 5.0 (GraphPad Software, La Jolla, CA). Statistical analysisin form of Student's t-test or one-way analysis of variance (ANOVA),was considered to be significant when $\mathrm{p}$-values below 0.05 .

\section{Results}

\section{AGAP2-AS1 was enriched in BC cell-secreted exosomes}

Previously, we identified an upregulation of AGAP2-AS1 in trastuzumab-resistant BC cells compared to parental cells [17]. However, whether AGAP2-AS1 can be packaged into extracellular materials, such as exosome, is not well known. By using SKBR-3-TR cells, we extracted exosomes from thecell culture medium and confirmed by transmission electron microscopy (TEM) (Fig. 1A) and NanoSight analysis (Fig. 1B). The exosomes were further confirmed by western blotting analysis of exosomal markers, TSG101 and HSP70 (Fig. 1C). qRT-PCR indicated that the expression of AGAP2-AS1 packaged in exosome was significantly increased in trastuzumab-resistant cells in contrast to the respective parental cells (Fig. 1D). Moreover, AGAP2-AS1 was more enriched in exosomes in contrast to intracellular portions, indicating that AGAP2-AS1 may function via incorporating into exosomes (Fig. 1E). Importantly, knockdown of cellular AGAP2-AS1 via transfection of AGAP2-AS1 small interfere vectors induced a dramatically decrease of exosomal AGAP2-AS1 expression (Fig. 1F and 1G), while overexpression of cellular AGAP2-AS1 showed opposite effects (Fig. 1H and 1I), strengthening our conclusion that AGAP2AS1 was enriched in extracellular exosomes.

\section{Exosomal AGAP2-AS1 was internalized by BC parental cells to induce trastuzumab resistance}

To directly probe exosomal AGAP2-AS1 induces trastuzumab resistance in recipient cells, we incubated SKBR-3 and BT474 cells with exosomes derived from SKBR-3-TR cells. As shown in Fig. 2A, SKBR-3-TRsecreted exosomes significantly suppressed trastuzumab-induced cytotoxicity. However, treatment with exosomes isolated from SKBR-3-TR cells which are silenced with AGAP2-AS1 lost the ability to induce trastuzumab resistance of parental cells (Fig. 2B). On the other hand, incubation SKBR-3 and BT474 cells with AGAP2-AS1-enriched SKBR-3-TR-derived exosomes significantly increased the cellular expression of AGAP2-AS1 of recipient cells while AGAP2-AS1-silenced exosomes showed no influence on AGAP2-AS1 expression level (Fig. 2C), suggesting that exosomal AGAP2-AS1 may be internalized to induce trastuzumab resistance.

To provide direct evidence that exosomal AGAP2-AS1 was internalized by BC cells, we labeled extracted exosomes with PKH67 green dye. As expected, a strong green fluorescence signal was observed in 
recipient cells while no signal was observed in PBS control group (Fig. 2D). To eliminate the possibility that endogenous AGAP2-AS1 induced trastuzumab resistance of BC parental cells. We built AGAP2-AS1knockout SKBR-3 and BT474 cells from using the CRISPR-Cas9 approach with double-strained sgRNAs targetingAGAP2-AS1. A successful deficiency of AGAP2-AS1 was confirmed by our qRT-PCR analysis (Fig. 2E). CCK8 assay proved that AGAP2-AS1-overexpressed cell-secreted exosomes promoted trastuzumab resistance of AGAP2-AS1-knockout cells (Fig. 2F). These results were consistent with that of AGAP2-AS1-wild type BC cells (Fig. 2G). Taken together, we demonstrated that AGAP2-AS1overexpression cells could spread trastuzumab resistance by transferring exosomal AGAP2-AS1 to sensitive cells.

\section{Exosomal AGAP2-AS1 promotes trastuzumab resistance via inducing autophagy}

It is well demonstrated that autophagy may decrease the cytotoxicity ofdrugsupon cancer cells, inducing resistance[20]. Interestingly, the Gene Ontology (GO) analysis showed that autophagy may be involved in the function of AGAP2-AS1 (Fig. 3A). To find whether AGAP2-AS1 regulates trastuzumab resistance via inducing autophagy, we firstly measured the autophagy activity of $B C$ cells. Remarkably, compared to BC parental cells, trastuzumab-resistant cells showed higher activity of autophagy, as evidenced by increased expression of LC3-II, decreasedexpression of p62 protein and increased formation of LC3 puncta, than the corresponding parental cells, confirming the close association between autophagy and drug resistance in cancer (Fig. 3B-C). To identify the direct role of autophagy in trastuzumab resistance, we used chloroquine (CQ), a well-known autophagy inhibitor[21]. As expected, CQ treatment inhibited autophagy activity and deteriorated trastuzumab resistance (Fig. 3D-E).

We then explored whether AGAP2-AS1 influence autophagy of BC cells. Compared to control group, knockdown of AGAP2-AS1 caused decreased LC3-II, elevated p62 expression and reduced number of LC3 puncta in SKBR-3-TR and BT474-TR cells (Fig. 3F-G). Importantly, an increased autophagy activity was observed in SKBR-3 and BT474 cells when incubated with exosomes derived from SKBR-3-TR cells, however, the pro-autophagic effect was not observed when we incubated with exosomes derived from siAGAP2-AS1-transfected SKBR-3-TR cells (Fig. 3H-I). Collectively, we proved that exosomal AGAP2-AS1 transmit trastuzumab resistance via regulating autophagy activity.

\section{Exosomal AGAP2-AS1 upregulates ATG10 expression to induce trastuzumab resistance in vitro}

To clarify the functional target of AGAP2-AS1 in autophagy and trastuzumab resistance, we performed RNA sequencing with AGAP2-AS1 knockout SKBR-3 cellsandwild-type controlled cells. Preliminarily, 1247 genes were identified with statistical significance (Fold change $>2$ ) in both cell lines (Fig. 4A). Given the fact that AGAP2-AS1 may influence trastuzumab resistance via regulating autophagy, we focus on 27 autophagy-related genes, including ATG10 and ATG5 (Fig. 4B). By performing qRT-PCR, we identified five genes were significantly altered upon silence of AGAP2-AS1 in trastuzumab resistant cells (Fig. 4C). Western blotting showed that the protein levels of two of those genes, ATG10 and ATG5 were silenced by AGAP2-AS1 knockdown (Fig. 4D). Moreover, ATG10 was upregulated in trastuzumabresistant cells compared to parental cells in both transcript and protein levels (Fig. 4E), whereas ATG5 
was not altered (data not shown). Functionally, knockdown of ATG10 significantly reversed the AGAP2AS1-induced autophagy activity and trastuzumab resistance (Fig. 4F and G), while silence of ATG5 showed no effect (data not shown), suggesting that ATG10 may be directly targeted by AGAP2-AS1 and mediates the AGAP2-AS1-induced autophagy and trastuzumab resistance.

\section{Exosomal AGAP2-AS1 promotes trastuzumab resistance via ATG10 in vivo}

To further confirm the essential role of exosomal AGAP2-AS1 in autophagy and trastuzumab resistance, we established xenografts in BALB/c nude mice models. Mice were inoculated subcutaneously withSKBR-3 cells and randomly divided into three groups. Mice of each group received PBS, SKBR-3-TREXO $_{\text {Vector, }}$ or SKBR-3-TR-EXO ${ }_{\text {AGAP2-AS1 }}$ treatments accordingly,onceevery 3 days for 5 weeks (Fig. 5A). Meanwhile, each group received intraperitoneal treatment of trastuzumab once every two days for 4 weeks (Fig. 5B). Mice treated with SKBR-3-TR-EXO ${ }_{\text {AGAP2-AS1 }}$ showed increased growth of xenografts, greater size and weight compared to PBS and SKBR-3-TR-EXO Vector-treated mice (Fig. 5C-G), suggesting that exosomal AGAP2-AS1 increased trastuzumab resistance of mice. Moreover, IHC analysis showed that SKBR-3-TR-EXO ${ }_{\text {AGAP2-AS1 }}$ treatmentincreased ATG10 expression compared to PBS and SKBR-3-TREXO $_{\text {Vector }}$ groups $\left(\right.$ Fig. $5 \mathrm{H}$ ). Consistently, tumors in SKBR-3-TR-EXO AGAP2-AS1 $_{1}$ groupshowed increased formation of LC3 puncta (Fig. 5I). These results suggest that exosomal AGAP2-AS1 induced trastuzumab resistance via promoting ATG10 expression and autophagy in vivo.

\section{AGAP2-AS1 was interacted and stabilized by ELAVL1}

To verify the RNA binding protein of AGAP2-AS1, we performed RNA pulldown assay usingbiotinylated AGAP2-AS1 and sense control. An obvious band of 35-50 kDa was identified with clear significance between AGAP2-AS1 and sense control (Fig. 6A). According to mass spectrometry analysis and western blotting after RNA pulldown, we identified it was embryonic lethal abnormal version like RNA binding protein 1 (ELAVL1) (Fig. 6B-D). Consistently, RNA-FISH verified that AGAP2-AS1 and ELAVL1 colocalized mostly in the cytoplasm of SKBR-3 cells (Fig. 6E). RIP assay further confirmed the direct interaction between AGAP2-AS1 and ELAVL1 (Fig. 6F). Furthermore, serial deletion analysis revealed that the 900-1200 nt region of AGAP2-AS1 was essential for binding with ELAVL1 (Fig. 6G). Prediction by POSTAR2 (http://lulab.life.tsinghua.edu.cn/postar/index.php) showed a binding motif of ELAVL1 at the 937-957 nt region of AGAP2-AS1 (Fig. 6H). According to RNAfold, an online software predicting spatial structure[22], the potential binding area formed stem-loop structure and showed MFE(Fig. 6I), which strongly suggests a direct binding.

It is reported that ELAVL1 play a role in stabilizing mRNAs by binding AU-rich elements (AREs) [23]. To verify whether ELAVL1 stabilized AGAP2-AS1, we silenced ELAVL1 expression in BC cells (Fig. 6J). Silence of ELAVL1 caused downregulation of AGAP2-AS1 in trastuzumab resistant cells (Fig. 6K). Importantly, when we blocked RNA transcription process by using actinomycin D (ActD), the stability of AGAP2-AS1 RNA wasdramatically suppressed in ELAVL1-silenced cells compared to that in control cells (Fig. 6L). Our results indicate that ELAVL1 directly binds to AGAP2-AS1 and maintain its stability. 
AGAP2-AS1- ELAVL1 complex promote H3K4 trimethylation and H3K27 acetylation at theATG10 promoter

By analyzing the transcriptional modification region at $h t t p: / / g e n o m e . u c s c . e d u /$, we found potential H3K4 trimethylation (H3K4me3) and H3K27 acetylation (H3K27ac) binding areas at ATG10 promoter region (Fig. 7A). To probe AGAP2-AS1-ELAVL1 complex increases ATG10 expression by transcription activation, we performed ChIP assay. As expected, both H3K4me3 and H3K27ac enrichment were identified at ATG10 promoter region (Fig. 7B). Moreover, ELAVL1 was also enriched at ATG10 promoter region (Fig. 7C).

To further confirm AGAP2-AS-ELAVL1 interacted with ATG10 promoter to induce histone modification, we evaluated the role of AGAP2-AS1 and ELAVL1 in H3K27me3 and H3K27ac enrichment. Fig. 7D showed that knockdown of AGAP2-AS1 decreased H3K27me3 and H3K27ac enrichment in SKBR-3-TR and BT474-TR cells. Enrichment of the ATG10 promoter sequences associated with H3K4me3 and H3K27ac were increased in SKBR-3 and BT474 cells incubated with exosomes secreted by AGAP2-AS1-transfected SKBR-3-TR cells (Fig. 7E). Interestingly, the effect caused by ectopic expression of AGAP2-AS1 was significantly reversed in cells silenced with ELAVL1, indicating that ELAVL1 is essential for AGAP2-AS1induced transcription activation (Fig. 7F). Although ELAVL1 could participate in the regulation of RNA stability, ActD treatment showed that ELAVL1 had little effect on half-life of ATG10 mRNA (data not shown).

\section{Targeting AGAP2-AS1 with ASO showed potential inreversing trastuzumab resistance}

Various in vitro, in vivo and pre-clinical studies proved that antisense oligonucleotide (ASO) may serving as promising small molecular drugs by generating specific vectors against diverse RNAs[24]. To investigate the potential of ASOs targeting AGAP2-AS1 in overcoming trastuzumab resistance, three ASOs against AGAP2-AS1 and one negative control were designed (Fig. 8A). AGAP2-AS1 was silenced by ASO-1 and ASO-2 in trastuzumab resistant BC cells (Fig. 8B). Cell suppression induced by trastuzumab was promoted by ASO-interfered AGAP2-AS1 (Fig. 8C-D). ATG10 expression and autophagy activity were also suppressed when AGAP2-AS1 was silenced (Fig. 8E). By optimizing the ASOs, we tested their functional efficiency in vivo. Free uptake assay revealed that two ASOs inhibited AGAP2-AS1 expression compared to negative control in SKBR-3-TR cells in adose-dependent manner (Fig. 8F). SKBR-3-TR cells were planted into mammary fat pads. When orthotopic xenograft tumor was established, mice were separated into 3 groups and injected via tail vein with ASO-1, ASO-2 or negative control ASO twice a week for 4 weeks (Fig. 8G). Meanwhile, each group received intraperitoneal treatment of trastuzumab. Tumor growth was significantly suppressed in two ASO-treated groups compared to ASO-control group, suggesting that trastuzumab combined with AGAP2-AS1 knockdown via ASO could induce better anticancer effects, when compared to trastuzumab treatment only (Fig. 8H). ATG10 expression was also suppressed in tissues treated with the two ASOs compared to control group (Fig. 8I). Taken together, our results indicate that targeting AGAP2-AS1 with ASOcombing drugs might be a promising therapeutic approach to overcome trastuzumab resistance. 


\section{Circulating exosomal AGAP2-AS1 predicts response to trastuzumab treatment of BC patients}

To determine the predictive value of exosomal AGAP2-AS1 in trastuzumab response of BC patients, we analyzed its expression in 90 serum samples from patients receiving trastuzumab treatment (45 responding and 45 non-responding patients). As shown in Fig. 9A, exosomal AGAP2-AS1 is upregulated in nonresponding patients compared to responding patients. Moreover, receiver operator characteristic (ROC) curve showed a relatively high predictive value of exosomal AGAP2-AS1 in discriminating responding and non-responding patients, with an area under the curve (AUC) at 0.784 , and diagnostic sensitivity and specificity at $77.8 \%$ and $73.3 \%$, respectively (Fig. 9B). By stratifying patients into a high or low exosomal AGAP2-AS1 expression group with a stratification criteria (4.06) obtained from the ROC curve, we found that the percent of patients showing response to trastuzumab therapy was much lower in high AGAP2-AS1-expressed group than that fromlow AGAP2-AS1-expressed group (Fig. 9C), further strengthening the predictive value of exosomal AGAP2-AS1 in discriminating responding and nonresponding patients.

\section{Discussion}

Despite current chemotherapeutic regimens that evidently improved the survival of metastatic tumors, nearly all HER-2+ BC patients finally develop into resistance[25, 26].LncRNAs are dysregulated invarious cancers, and modulates drug resistance byinteracting with RNA and proteins[27]. Until now, the precise regulatory mechanisms by which IncRNAs regulate trastuzumab resistance in BC is still not clearlydefined. In this study, we investigated the pivotal role and post-transcriptional regulation of IncRNA AGAP2-AS1 in trastuzumab resistance. We revealed that AGAP2-AS1 promotes trastuzumab resistance via packaging into exosomes. In addition, AGAP2-AS1 directly interacted with ELAVL1 which in turn stabilizes AGAP2-AS1; the AGAP2-AS1-ELAVL1 complex could directly bind to promoter of ATG10, inducing an enhanced transcriptional activity of ATG10through increasing H3K4me3 and H3K27acenrichment, and finally lead to the activation autophagy and trastuzumab resistance (Fig. 9D).

During recent years, more useful therapeutic methods were developed for the treatment of HER-2+ breast cancer [28], however, trastuzumab resistance still occurs and dramatically reduced the clinical practicality and response, which has become one of the most urgent challenges for promoting breast cancer outcome. Furthermore, lacking of effective prognostic biomarkers that could be used for predicting therapeutic response to trastuzumab also greatly hindered the improvement of HER-2 positive patients [29]. In addition to the molecular alterations discussed above, there is increased expression of a number of RTKs-IGF-1Rß, c-Met, and EGFR-that are implicated in trastuzumab resistance [30]. Currently, very limited biomarkers are useful in guiding therapeutic decisions among HER-2+ patients except for HER-2 itself [31]. Hence, more and more studies needs to be devote to finding promising therapeutic targets and prognostic biomarkers[32, 33].

In recent years, the functions of IncRNAs have drawn more and more attention. A great number of studies have indicated that IncRNAs play important roles in all steps of carcinogenesis and tumor progression, 
and IncRNAs could be used as new prognostic markers [34,35]. More recently, emerging evidence has indicated that exosomes regulate malignancyby transferring multiple classes of cargo molecules to recipient cells to mediateintercellular communication, including IncRNAs. Exosomal IncRNAs participate in tumor formation,proliferation, metastasis and chemotherapy resistance by regulating oncogenes ortumor suppressor genes $[9,36,37]$. However, communication in BC cells withdifferent drug response via exosomes is obscured. In this study, we proved that AGAP2-AS1 IncRNA could be incorporated into exosomes and further internalized by recipient cells to transmit resistant property.

Autophagy can be a double-edged sword for resistant tumors: it participates in the development of drug resistance and protects cancer cells from chemotherapeutics but can also kill cancer cells in which apoptosis pathways are inactive. Therefore, research on the regulation of autophagy to combat resistance is expanding and is becoming increasingly important[38]. By screening potential targeted mRNAs, we identified ATG10 as one potentially regulated by AGAP2-AS1 and may play pivotal roles in trastuzumab resistance via regulating autophagy. Further experimental data proved that ATG10 expression is positively regulated by AGAP2-AS1 and essential for AGAP2-AS1-induced autophagy and trastuzumab resistance. ATG10 is an autophagic E2-like enzyme that interacts with ATG7 to recruit ATG12 and modulates the conversion of LC3-I to LC3-II [39]. Thus, ATG10 plays a critical role in autophagosome formation. Emerging evidence has emphasized that ATG10 displayed higher expression level in tumors of malignancies such as CRC [40] and lung cancer [41]. In addition, increased expression of ATG10 is positively linked with lympho-vascular invasion and predicts decreased overall survival times [42].

Another finding is the identification of the RBP, ELAVL1 (also known as Hu Antigen R, HuR), which binds to AGAP2-AS1 and regulates stability. ELAVL1 is a member of the RBP ELAV (embryonic lethal abnormal version) family, well studied for its role in RNA splicing and mRNA post-transcriptional regulation [43]. Various studies reported the critical role of ELAVL1 in cancer initiation, progression and resistance, via stabilizing targeted mRNAs. For example, Luo et al. revealed that ZEB1-AS1 IncRNA interacts with ELAVL1, thereby maintaining stability of ZEB1 mRNA in breast cancer [44]. Another study by Chen et al. found that IncRNA HOXB-AS1 promotes cell growth in multiple myeloma via FUT4 mRNA stability by ELAVL1 [45]. Our results suggest that there exists a direct interaction between AGAP2-AS1 and ELAVL1 protein, and the binding of AGAP2-AS1 by ELAVL1 increased the stability of AGAP2-AS1. In addition, AGAP2-AS1-ELAVL1 complex further binds to the promoter of ATG10, altering histone modifications and activating transcription. Moreover, the AGAP2-AS1-induced formation of H3K27ac and H3K4me3 was abolished by knockdown of ELAVL1, suggesting that ELAVL1 is essential for this epigenetic regulation. Reciprocally, overexpression of ELAVL1 only partlyreversed thedecrease of H3K4me3 and H3K27ac enrichment inAGAP2-AS1 silencing cells, indicating thatthe enhanced transcriptional activity at ATG10 promoter is dependent on the histone modification induced by AGAP2-AS1-ELAVL1 complex.

We need to point out two limitations of our study. First, we explored the experimental role of ASO targeting AGAP2-AS1 in reversing trastuzumab resistance, however, this may need further validation in clinical trials. Second, we preliminarily confirmed the function of AGAP2-AS1/ELAVL1/ATG10 in 
autophagy and trastuzumab resistance, however the precise regulatory model, and the ongoing interactions with other cellular factors in tumor microenvironment during this process need more meaningful studies.

\section{Conclusions}

In summary, we demonstrated the crucial role of AGAP2-AS1 in trastuzumab resistance of BC by epigenetically increasing ATG10 expression and thereby promoting autophagy. Our discovery not only help us get a better understanding the regulatory potential of AGAP2-AS1 in drug resistance of HER-2+ $\mathrm{BC}$, but alsouseful for finding promising drug targets and developing novel therapeutic strategiesto overcome resistance.

\section{List Of Abbreviations}

ASO: AntisenseOligonucleotides;

ATG10: Autophagy related 10;

ATCC: American Tissue Culture Collection;

ANOVA : Student's t-test or one-way analysis of variance;

BC: Breast cancer;

ChIP: Chromatin immunoprecipitation;

DAPI: 4',6-diamidino-2-phenylindole;

DMEM: Dulbecco's modified Eagle medium;

ELAVL1: Embryonic Lethal Abnormal Version Like RNA binding protein 1;

FBS: Fetal bovine serum;

FISH: Fluorescence in situ hybridization;

GAPDH: Glyceraldehyde 3-phosphate dehydrogenase;

HER-2: Human epidermal growth factor receptor 2;

IHC: Immunohistochemistry;

LncRNAs: Long noncoding RNAs;

mRNA: messenger RNA; 
MFE: minimum free energy;

NC: Negative control;

OS: Overall survival;

PCR: Polymerase chain reaction;

PVDF: Polyvinylidene fluoride;

PFS: Progressive-free survival;

PBS: phosphate-buffered saline;

RIP: RNA immunoprecipitation;

RBPs: RNA-binding proteins;

siRNA: small interfering RNA;

SD: Standard deviation;

TEM: Transmission electron microscopy.

\section{Declarations}

\section{Ethics approval and consent to participate}

This study was approved by Research Scientific Ethics Committee of The First Affiliated Hospital of Zhengzhou University, The First People's Hospital of Chenzhou City and Hainan General Hospital. All participants signed informed consent prior to using the tissues and serum samples for scientific research.

\section{Consent for publication}

Not applicable.

\section{Availability of data and material}

The datasets used and/or analyzed during the current study are available from the corresponding author on reasonable request.

\section{Competing interests}

The authors declare that they have no competing interests.

\section{Funding}


This study is supported by National Science Foundation of China (81960475) and The "Nanhai series" talent cultivation program of Hainan Province.

Authors' contributions

Huaying Dong,Mingli Han,Hongbo Qu,andXueke Qian mainly did the experiment and wrote the paper. Hui Cao, Xiangke Li, Dongwei Dou, Xue Yang, Yunqing Yang, Na Han, Mulin Ye, Jing Han, Fan Zhang, Wei Wang helped do the cell experiment. Yichao Dingand Mingwei Xie did the statistical work.

\section{Acknowledgements}

We thank Professor Manran Liu, The Key Laboratory of Laboratory Medical Diagnostics, Chinese Ministry of Education, Chongqing Medical University, Chongqing 400016, China, for critical technical support.

\section{References}

1. Boero S, Morabito A, Banelli B, Cardinali B, Dozin B, Lunardi G, Piccioli P, Lastraioli S, Carosio R, Salvi $S$ et al: Analysis of in vitro ADCC and clinical response to trastuzumab: possible relevance of FcgammaRIIIAFcgammaRIIA gene polymorphisms and HER-2 expression levels on breast cancer cell lines. Journal of translational medicine 2015, 13:324.

2. Bighin C, Pronzato P, Del Mastro L: Trastuzumab emtansine in the treatment of HER-2-positive metastatic breast cancer patients. Future oncology 2013, 9(7):955-957.

3. Amadori D, Milandri C, Comella G, Saracchini S, Salvagni S, Barone C, Bordonaro R, Gebbia V, Barbato A, Serra $\mathrm{P}$ et al: A phase I/II trial of non-pegylated liposomal doxorubicin, docetaxel and trastuzumab as first-line treatment in HER-2-positive locally advanced or metastatic breast cancer. European journal of cancer 2011, 47(14):2091-2098.

4. Herrera-Solorio AM, Armas-Lopez L, Arrieta O, Zuniga J, Pina-Sanchez P, Avila-Moreno F: Histone code and long non-coding RNAs (IncRNAs) aberrations in lung cancer: implications in the therapy response. Clinical epigenetics 2017, 9:98.

5. Li Z, Qian J, Li J, Zhu C: Knockdown of IncRNA-HOTAIR downregulates the drug-resistance of breast cancer cells to doxorubicin via the PI3K/AKT/mTOR signaling pathway. Experimental and therapeutic medicine 2019, 18(1):435-442.

6. Arshi A, Sharifi FS, Khorramian Ghahfarokhi M, Faghih Z, Doosti A, Ostovari S, Mahmoudi Maymand E, Ghahramani Seno MM: Expression Analysis of MALAT1, GAS5, SRA, and NEAT1 IncRNAs in Breast Cancer Tissues from Young Women and Women over 45 Years of Age. Molecular therapy Nucleic acids 2018, 12:751-757.

7. Zhu HY, Bai WD, Ye XM, Yang AG, Jia LT: Long non-coding RNA UCA1 desensitizes breast cancer cells to trastuzumab by impeding miR-18a repression of Yes-associated protein 1. Biochemical and biophysical research communications 2018, 496(4):1308-1313. 
8. Shi SJ, Wang LJ, Yu B, Li YH, Jin Y, Bai XZ: LncRNA-ATB promotes trastuzumab resistance and invasion-metastasis cascade in breast cancer. Oncotarget 2015, 6(13):11652-11663.

9. Han M, Gu Y, Lu P, Li J, Cao H, Li X, Qian X, Yu C, Yang Y, Yang X et al: Exosome-mediated IncRNA AFAP1-AS1 promotes trastuzumab resistance through binding with AUF1 and activating ERBB2 translation. Molecular cancer 2020, 19(1):26.

10. Pereira B, Billaud M, Almeida R: RNA-Binding Proteins in Cancer: Old Players and New Actors. Trends in cancer $2017,3(7): 506-528$.

11. Upadhyay R, Sanduja S, Kaza V, Dixon DA: Genetic polymorphisms in RNA binding proteins contribute to breast cancer survival. International journal of cancer 2013, 132(3):E128-138.

12. Huang G, Song C, Wang N, Qin T, Sui S, Obr A, Zeng L, Wood TL, LeRoith D, Li M et al: RNA-binding protein CUGBP1 controls the differential INSR splicing in molecular subtypes of breast cancer cells and affects cell aggressiveness. Carcinogenesis 2020.

13. Zhou XJ, Wu J, Shi L, Li XX, Zhu L, Sun X, Qian JY, Wang Y, Wei JF, Ding Q: PTEN expression is upregulated by a RNA-binding protein RBM38 via enhancing its mRNA stability in breast cancer. Journal of experimental \& clinical cancer research : CR 2017, 36(1):149.

14. Ueno T, Masuda N, Kamigaki S, Morimoto T, Saji S, Imoto S, Sasano H, Toi M: Differential Involvement of Autophagy and Apoptosis in Response to Chemoendocrine and Endocrine Therapy in Breast Cancer. JBCRG-07TR. International journal of molecular sciences 2019, 20(4).

15. Yu T, Guo F, Yu Y, Sun T, Ma D, Han J, Qian Y, Kryczek I, Sun D, Nagarsheth N et al: Fusobacterium nucleatum Promotes Chemoresistance to Colorectal Cancer by Modulating Autophagy. Cel/ 2017, 170(3):548-563 e516.

16. Islam Khan MZ, Tam SY, Law HKW: Autophagy-Modulating Long Non-coding RNAs (LncRNAs) and Their Molecular Events in Cancer. Frontiers in genetics 2018, 9:750.

17. Dong H, Wang W, Mo S, Chen R, Zou K, Han J, Zhang F, Hu J: SP1-induced IncRNA AGAP2-AS1 expression promotes chemoresistance of breast cancer by epigenetic regulation of MyD88. Journal of experimental \& clinical cancer research : CR 2018, 37(1):202.

18. Seymour L, Bogaerts J, Perrone A, Ford R, Schwartz LH, Mandrekar S, Lin NU, Litiere S, Dancey J, Chen A et al: iRECIST: guidelines for response criteria for use in trials testing immunotherapeutics. The Lancet Oncology 2017, 18(3):e143-e152.

19. Dong H, Hu J, Zou K, Ye M, Chen Y, Wu C, Chen X, Han M: Activation of LncRNA TINCR by H3K27 acetylation promotes Trastuzumab resistance and epithelial-mesenchymal transition by targeting MicroRNA-125b in breast Cancer. Molecular cancer 2019, 18(1):3.

20. Liu L, Yan L, Liao N, Wu WQ, Shi JL: A Review of ULK1-Mediated Autophagy in Drug Resistance of Cancer. Cancers 2020, 12(2).

21. Xu R, Ji Z, Xu C, Zhu J: The clinical value of using chloroquine or hydroxychloroquine as autophagy inhibitors in the treatment of cancers: A systematic review and meta-analysis. Medicine 2018, 97(46):e12912. 
22. Lorenz R, Bernhart SH, Honer Zu Siederdissen C, Tafer H, Flamm C, Stadler PF, Hofacker IL: ViennaRNA Package 2.0. Algorithms for molecular biology : AMB 2011, 6:26.

23. Bakheet T, Hitti E, Al-Saif M, Moghrabi WN, Khabar KSA: The AU-rich element landscape across human transcriptome reveals a large proportion in introns and regulation by ELAVL1/HuR. Biochimica et biophysica acta Gene regulatory mechanisms 2018, 1861(2):167-177.

24. Sewing S, Gubler M, Gerard R, Avignon B, Mueller Y, Braendli-Baiocco A, Odin M, Moisan A: GaINAc Conjugation Attenuates the Cytotoxicity of Antisense Oligonucleotide Drugs in Renal Tubular Cells. Molecular therapy Nucleic acids 2019, 14:67-79.

25. Li N, Deng Y, Zhou L, Tian T, Yang S, Wu Y, Zheng Y, Zhai Z, Hao Q, Song D et al: Global burden of breast cancer and attributable risk factors in 195 countries and territories, from 1990 to 2017: results from the Global Burden of Disease Study 2017. Journal of hematology \& oncology 2019, 12(1):140.

26. Tian T, Wang M, Lin S, Guo Y, Dai Z, Liu K, Yang P, Dai C, Zhu Y, Zheng Y et al: The Impact of IncRNA Dysregulation on Clinicopathology and Survival of Breast Cancer: A Systematic Review and Metaanalysis. Molecular therapy Nucleic acids 2018, 12:359-369.

27. Huang P, Li F, Li L, You Y, Luo S, Dong Z, Gao Q, Wu S, Brunner N, Stenvang J: IncRNA profile study reveals the mRNAs and IncRNAs associated with docetaxel resistance in breast cancer cells. Scientific reports 2018, 8(1):17970.

28. Verma S, Miles D, Gianni L, Krop IE, Welslau M, Baselga J, Pegram M, Oh DY, Dieras V, Guardino E et al: Trastuzumab emtansine for HER2-positive advanced breast cancer. The New England journal of medicine 2012, 367(19):1783-1791.

29. Perez EA, de Haas SL, Eiermann W, Barrios CH, Toi M, Im YH, Conte PF, Martin M, Pienkowski T, Pivot $\mathrm{XB}$ et al: Relationship between tumor biomarkers and efficacy in MARIANNE, a phase III study of trastuzumab emtansine +/- pertuzumab versus trastuzumab plus taxane in HER2-positive advanced breast cancer. BMC cancer 2019, 19(1):517.

30. Gallardo A, Lerma E, Escuin D, Tibau A, Munoz J, Ojeda B, Barnadas A, Adrover E, Sanchez-Tejada L, Giner $D$ et al: Increased signalling of EGFR and IGF1R, and deregulation of PTEN/PI3K/Akt pathway are related with trastuzumab resistance in HER2 breast carcinomas. British journal of cancer 2012, 106(8):1367-1373.

31. Shetty P, Patil VS, Mohan R, D'Souza L C, Bargale A, Patil BR, Dinesh US, Haridas V, Kulkarni SP: Annexin A2 and its downstream IL-6 and HB-EGF as secretory biomarkers in the differential diagnosis of Her-2 negative breast cancer. Annals of clinical biochemistry 2017, 54(4):463-471.

32. Montemurro F, Prat A, Rossi V, Valabrega G, Sperinde J, Peraldo-Neia C, Donadio M, Galvan P, Sapino A, Aglietta $M$ et al: Potential biomarkers of long-term benefit from single-agent trastuzumab or lapatinib in HER2-positive metastatic breast cancer. Molecular oncology 2014, 8(1):20-26.

33. Ponde N, Bradbury I, Lambertini M, Ewer M, Campbell C, Ameels H, Zardavas D, Di Cosimo S, Baselga $\mathrm{J}$, Huober $\mathrm{J}$ et al: Cardiac biomarkers for early detection and prediction of trastuzumab and/or lapatinib-induced cardiotoxicity in patients with HER2-positive early-stage breast cancer: a NeoALTTO sub-study (BIG 1-06). Breast cancer research and treatment 2018, 168(3):631-638. 
34. Ghoveud E, Teimuri S, Vatandoost J, Hosseini A, Ghaedi K, Etemadifar M, Nasr Esfahani MH, Megraw TL: Potential Biomarker and Therapeutic LncRNAs in Multiple Sclerosis Through Targeting Memory B Cells. Neuromolecular medicine 2020, 22(1):111-120.

35. Storti CB, de Oliveira RA, de Carvalho M, Hasimoto EN, Cataneo DC, Cataneo AJM, De Faveri J, Vasconcelos EJR, Dos Reis PP, Cano MIN: Telomere-associated genes and telomeric IncRNAs are biomarker candidates in lung squamous cell carcinoma (LUSC). Experimental and molecular pathology 2020, 112:104354.

36. Kang M, Ren M, Li Y, Fu Y, Deng M, Li C: Exosome-mediated transfer of IncRNA PART1 induces gefitinib resistance in esophageal squamous cell carcinoma via functioning as a competing endogenous RNA. Journal of experimental \& clinical cancer research : CR 2018, 37(1):171.

37. Zhang W, Cai X, Yu J, Lu X, Qian Q, Qian W: Exosome-mediated transfer of IncRNA RP11838N2.4 promotes erlotinib resistance in non-small cell lung cancer. International journal of oncology 2018, 53(2):527-538.

38. Sun W, Li J, Zhou L, Han J, Liu R, Zhang H, Ning T, Gao Z, Liu B, Chen X et al: The c-Myc/miR-27b3p/ATG10 regulatory axis regulates chemoresistance in colorectal cancer. Theranostics 2020, 10(5):1981-1996.

39. Chang TC, Yu D, Lee YS, Wentzel EA, Arking DE, West KM, Dang CV, Thomas-Tikhonenko A, Mendell JT: Widespread microRNA repression by Myc contributes to tumorigenesis. Nature genetics 2008, 40(1):43-50.

40. Jo YK, Kim SC, Park IJ, Park SJ, Jin DH, Hong SW, Cho DH, Kim JC: Increased expression of ATG10 in colorectal cancer is associated with lymphovascular invasion and lymph node metastasis. PloS one 2012, 7(12):e52705.

41. Xie K, Liang C, Li Q, Yan C, Wang C, Gu Y, Zhu M, Du F, Wang H, Dai J et al: Role of ATG10 expression quantitative trait loci in non-small cell lung cancer survival. International journal of cancer 2016, 139(7):1564-1573.

42. Cao QH, Liu F, Yang ZL, Fu XH, Yang ZH, Liu Q, Wang L, Wan XB, Fan XJ: Prognostic value of autophagy related proteins ULK1, Beclin 1, ATG3, ATG5, ATG7, ATG9, ATG10, ATG12, LC3B and p62/SQSTM1 in gastric cancer. American journal of translational research 2016, 8(9):3831-3847.

43. Zhang J, Kong L, Guo S, Bu M, Guo Q, Xiong Y, Zhu N, Qiu C, Yan X, Chen Q et al: hnRNPs and ELAVL1 cooperate with uORFs to inhibit protein translation. Nucleic acids research 2017, 45(5):28492864.

44. Luo N, Zhang K, Li X, Hu Y: ZEB1 induced-upregulation of long noncoding RNA ZEB1-AS1 facilitates the progression of triple negative breast cancer by binding with ELAVL1 to maintain the stability of ZEB1 mRNA. Journal of cellular biochemistry 2020.

45. Chen R, Zhang X, Wang C: LncRNA HOXB-AS1 promotes cell growth in multiple myeloma via FUT4 mRNA stability by ELAVL1. Journal of cellular biochemistry 2019.

\section{Figures}


A

SKBR-3-TR
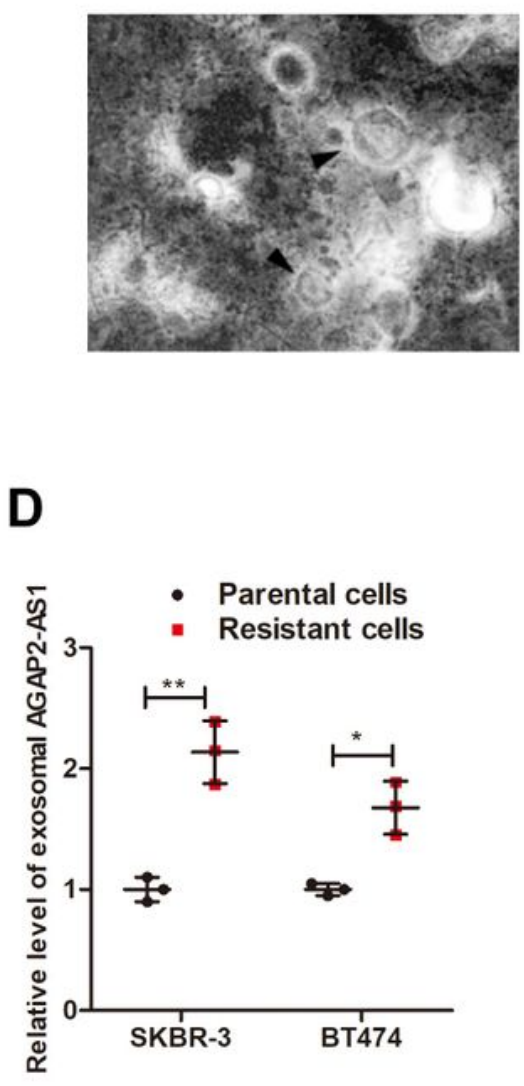

$\mathbf{E}$
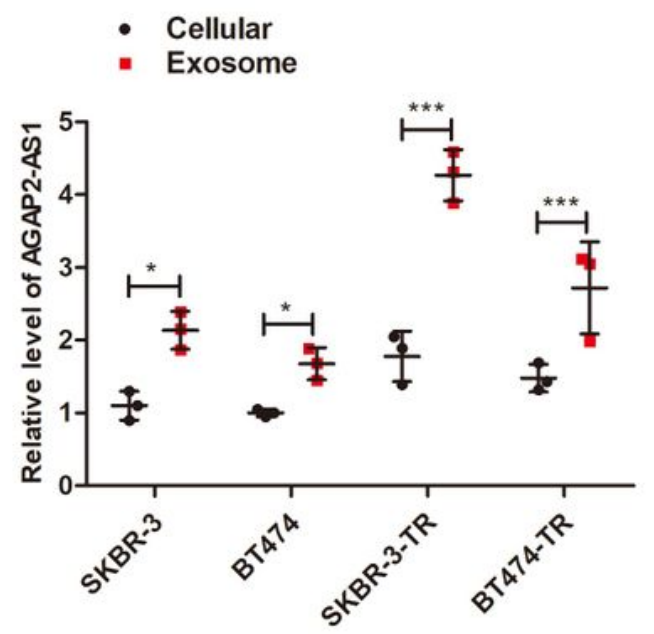

H
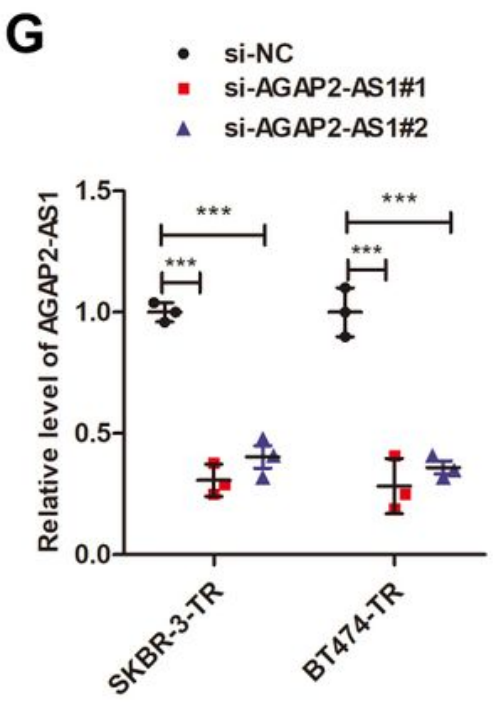

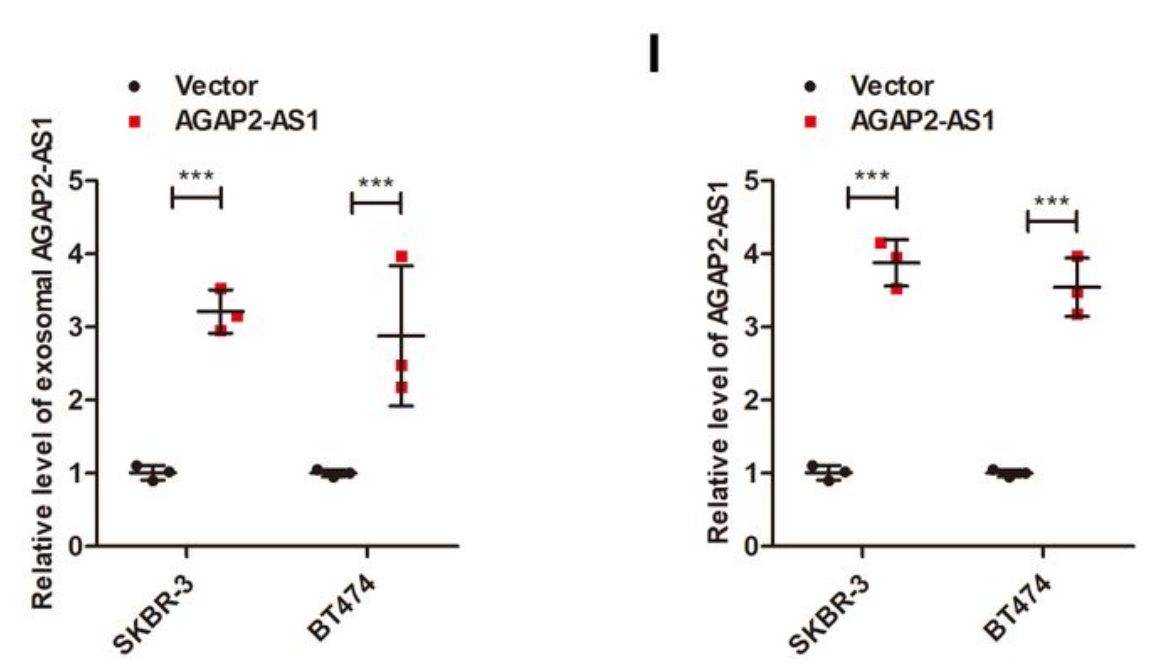

C

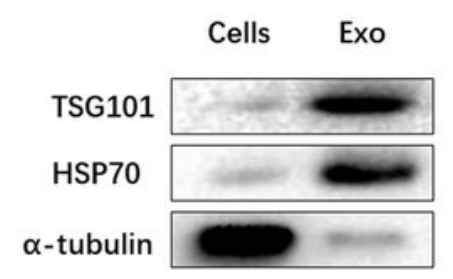

F $\quad: \begin{array}{ll}\text { si-NC } \\ \quad & \text { si-AGAP2-AS1\#1 } \\ & 4 \text { si-AGAP2-AS1\#2 }\end{array}$

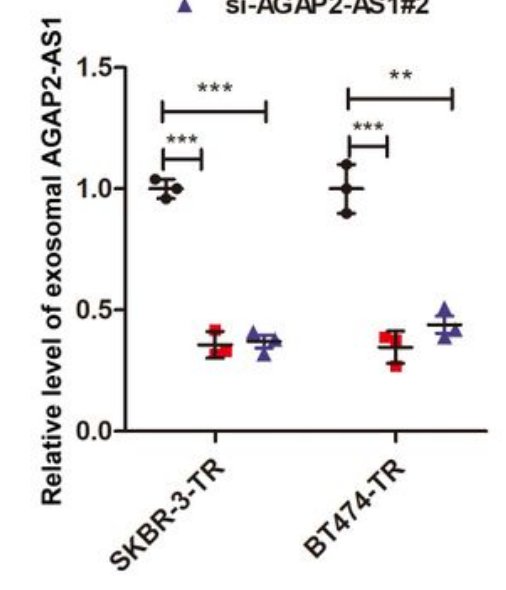

B

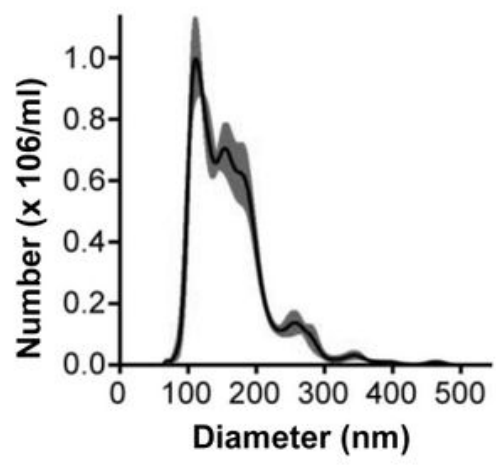

H

\section{Figure 1}

Extracellular AGAP2-AS1 was incorporated into exosomes. (A) Exosomes was identified under transmission electron microscopy. (B) NanoSight analysis showed the size distribution of extracellular vesicles. (C) Exosomal marker proteins were identified by western blot. (D) qRT-PCR was used to detect the expression of exosomal AGAP2-AS1 in sensitive and resistant breast cancer cells. (E) The expression of cellular or exosomal AGAP2-AS1 was determined by qRT-PCR. (F-G) Both total and exosomal AGAP2- 
AS1 were silenced by specific silencing vectors. $(\mathrm{H}-\mathrm{I})$ Both total and exosomal AGAP2-AS1 were overexpressed by transfection of overexpression vector. ${ }^{*} \mathrm{P}<0.05,{ }^{*} \mathrm{P}<0.01,{ }^{*} * * \mathrm{P}<0.001$

A

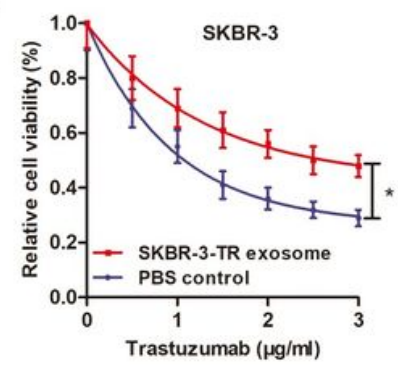

C

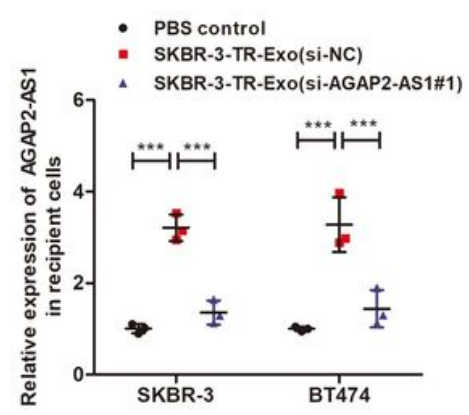

B

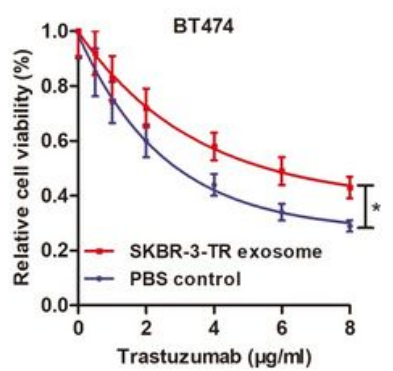

D

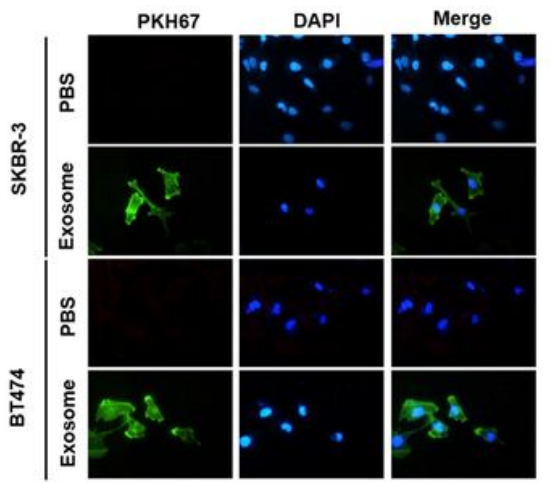

E

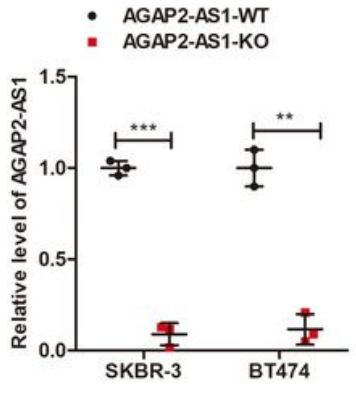

$\mathbf{F}$
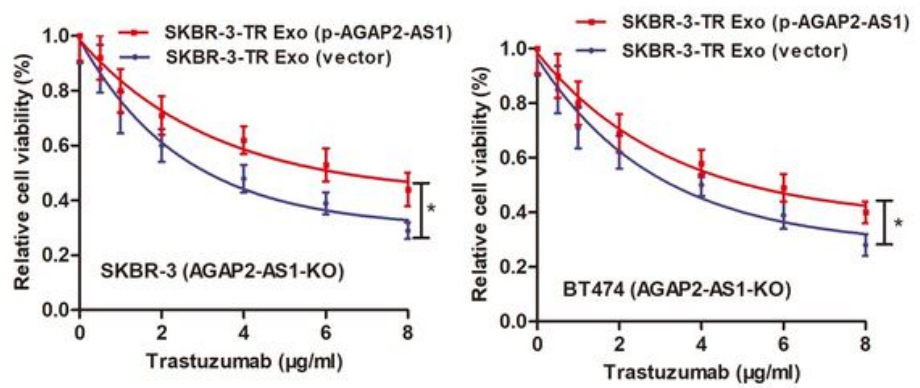

G

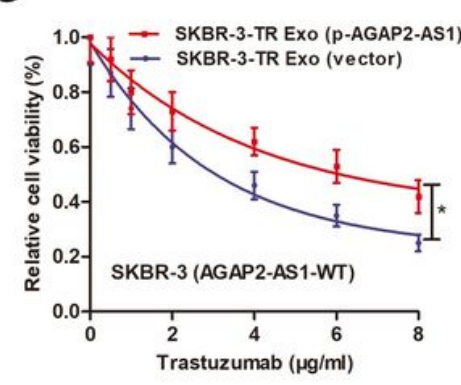

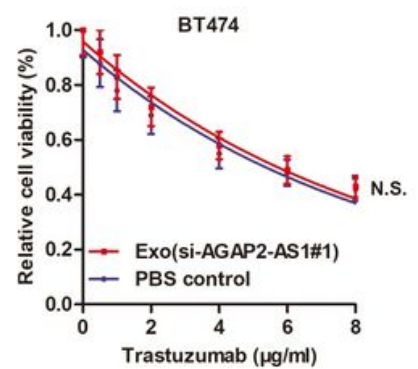

Trastuzumab $(\mu \mathrm{g} / \mathrm{ml})$

\section{Figure 2}

Exosomal AGAP2-AS1 was internalized by BC parental cells (A-B) Incubation of SKBR-3 and BT474 cells with SKBR-3-TR-secreted exosomes induced suppression of cytotoxicity induced by trastuzumab. However, this effect was not obvious when AGAP2-AS1 was silenced in SKBR-3-TR cells. (C) Incubation SKBR-3 and BT474 cells with AGAP2-AS1-enriched SKBR-3-TR-derived exosomes significantly increased the cellular expression of AGAP2-AS1 of recipient cells while AGAP2-AS1-silenced exosomes showed no influence on AGAP2-AS1 expression level. (D) A strong PKH67 fluorescence signal was observed in recipient cells while no signal was observed in PBS control group. (E) A successful deficiency of AGAP2AS1 was confirmed by qRT-PCR analysis. (F-G) CCK8 assay proved that AGAP2-AS1-overexpressed cellsecreted exosomes promoted trastuzumab resistance of AGAP2-AS1-knockout cells (F). These results were consistent with that of AGAP2-AS1-wild type BC cells $(G)$. ${ }^{*} P<0.05$ 
A

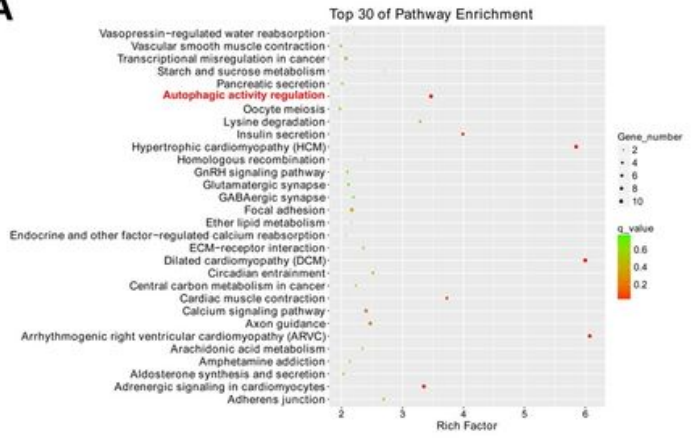

B

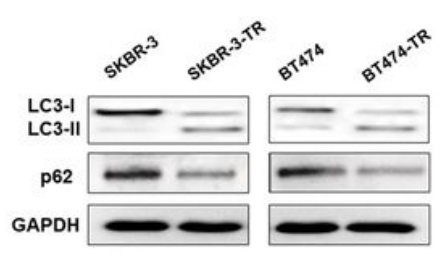

C

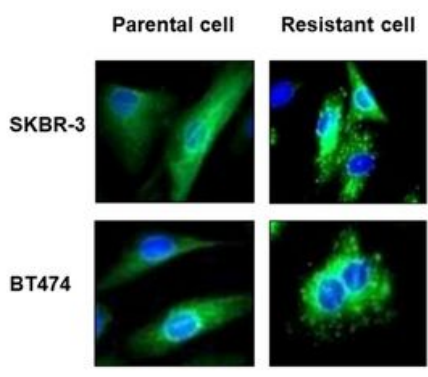

$\mathbf{F}$

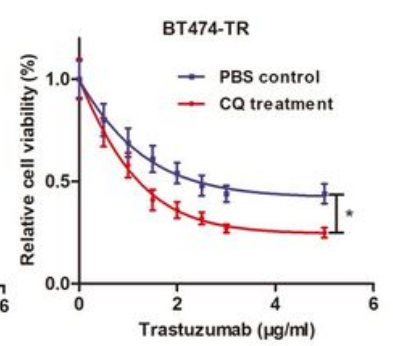

E

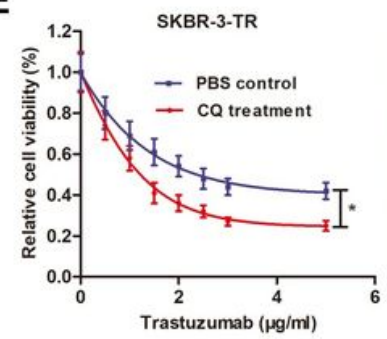

H

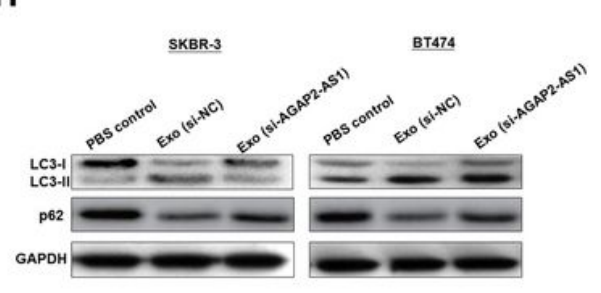

I

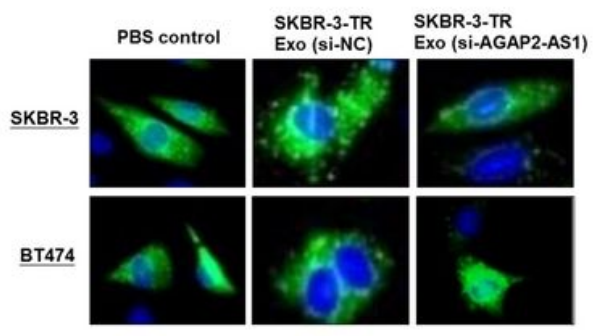

Figure 3

Exosomal AGAP2-AS1 promotes trastuzumab resistance via inducing autophagy (A) Gene Ontology analysis showed that autophagy may be involved in the function of AGAP2-AS1. (B-C) Trastuzumabresistant cells showed higher LC3-II, lower p62 protein levels (B) and increased formation of LC3 puncta (C) in contrast to sensitive cells. (D) Western blotting showed that autophagy inhibitor, CQ, significantly inhibited autophagy activity and deteriorated trastuzumab resistance. (E) CQ treatment deteriorated trastuzumab resistance. (F-G) Knockdown of AGAP2-AS1 caused decreased LC3-II, elevated p62 expression and reduced number of LC3 puncta in SKBR-3-TR and BT474-TR cells. (H-I) Incubation with SKBR-3-TR-derived exosomes increased autophagy activity of SKBR-3 and BT474 cells. However, the proautophagic effect was not observed when we incubated with exosomes derived from si-AGAP2-AS1transfected SKBR-3-TR cells. * $\mathrm{P}<0.05$ 
A

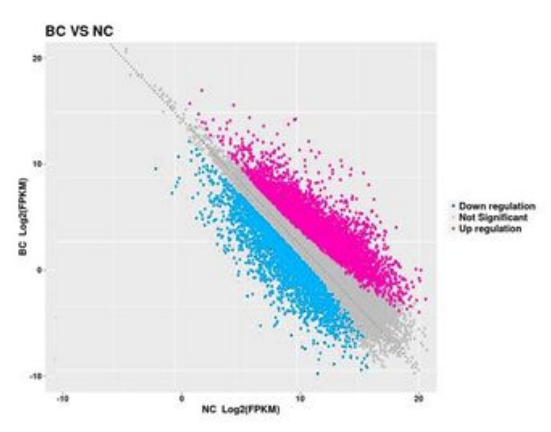

D

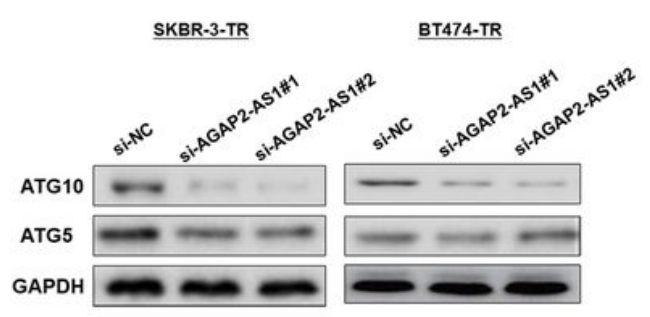

B

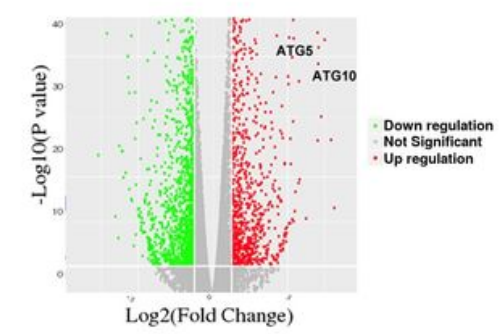

E

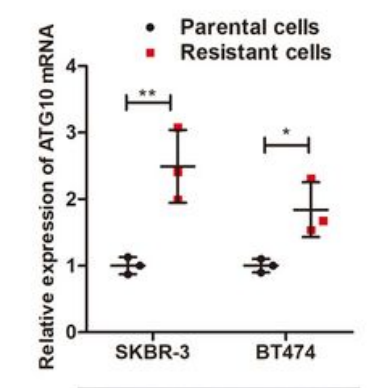

ATG10 $-\infty$

GAPDH

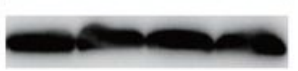

C

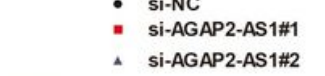

SKBR-3-TR

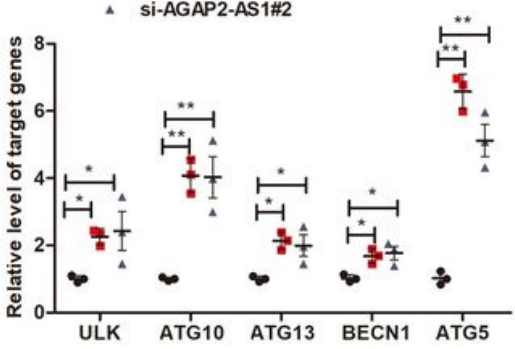

- si-NC

- si-AGAP2-AS1\#1

- Si-AGAP2-AS1\#2

BT474-TR

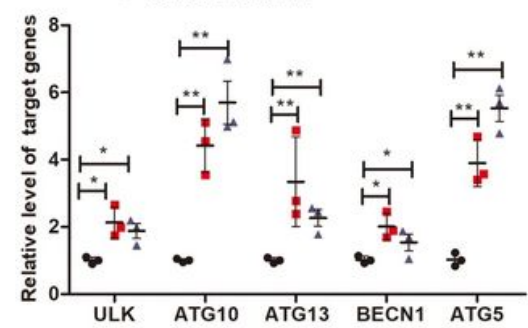

F

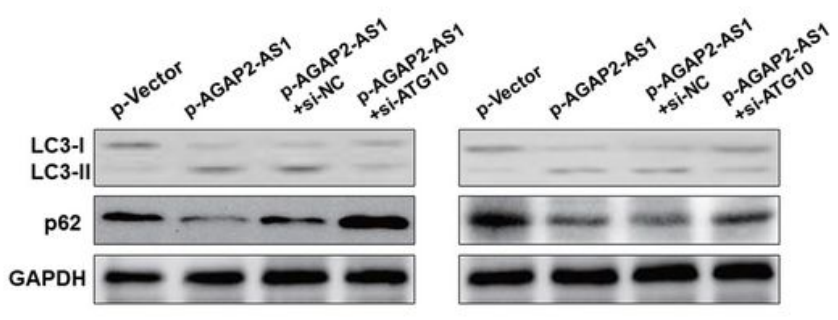

G

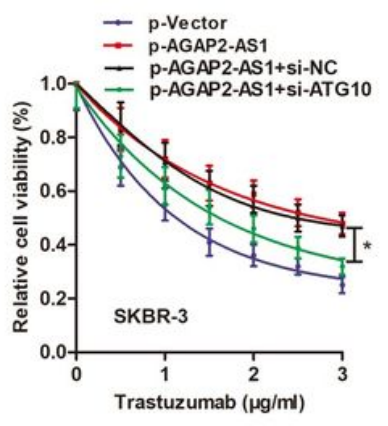

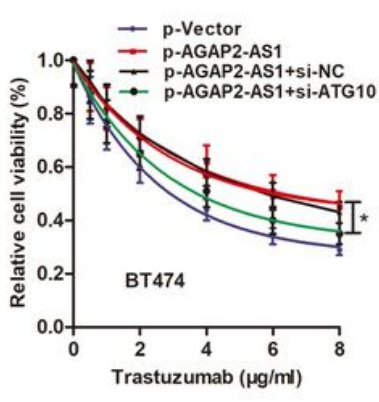

\section{Figure 4}

AGAP2-AS1 regulates trastuzumab resistance via upregulating ATG10 expression (A-B) Scatter plot and volcano plot showed the differentially expressed genes regulated by AGAP2-AS1, including 27 autophagyrelated genes, such as ATG10 and ATG5. (C) qRT-PCR verified five genes that were significantly altered upon knockdown of AGAP2-AS1. (D) Western blotting showed that knockdown of AGAP2-AS1 decreased ATG10 and ATG5 expression. (E) ATG10 was upregulated in trastuzumab-resistant cells compared to parental cells in both transcript and protein levels. (F-G) Knockdown of ATG10 significantly reversed the AGAP2-AS1-induced autophagy activity and trastuzumab resistance. ${ }^{*} \mathrm{P}<0.05,{ }^{*} \mathrm{P}<0.01$ 
A
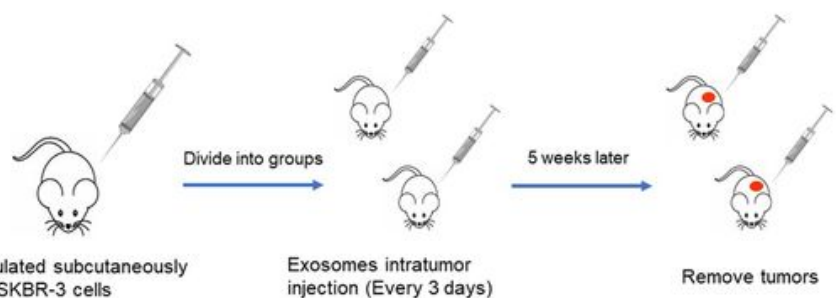
with SKBR-3 cells injection (Every 3 days)

Remove tumors

C

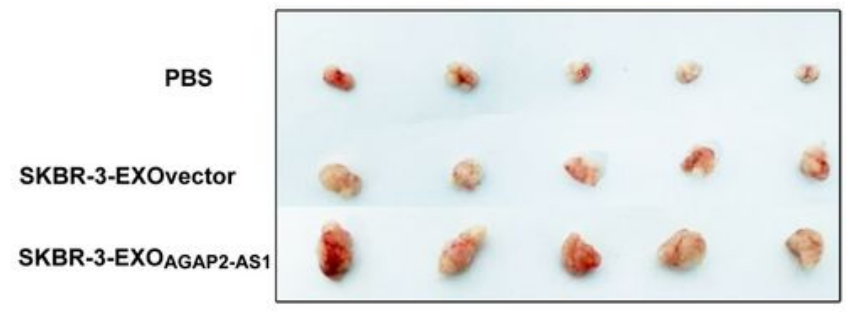

B

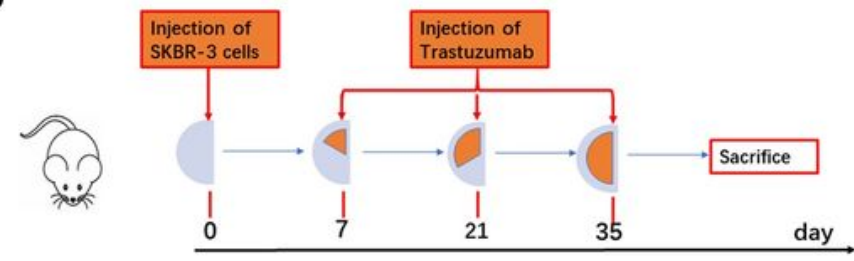

E

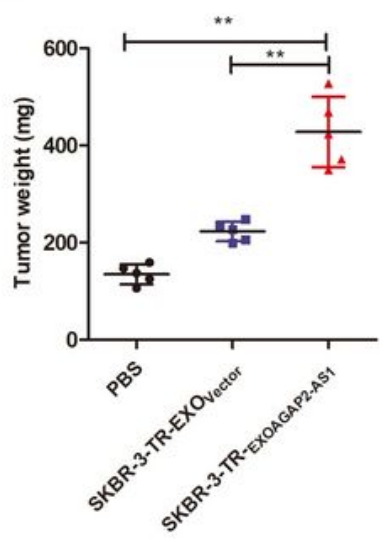

$\mathbf{H}$

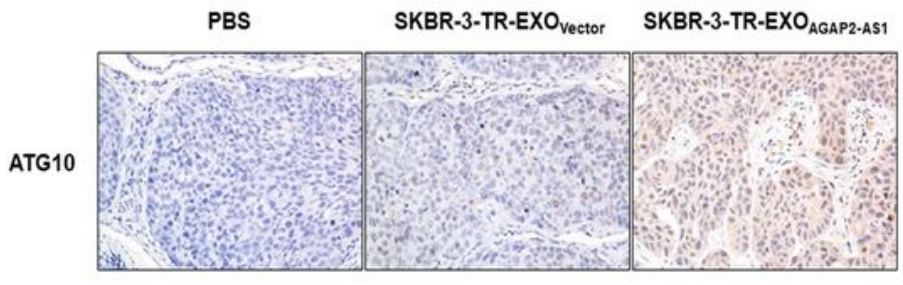

$\mathbf{F}$
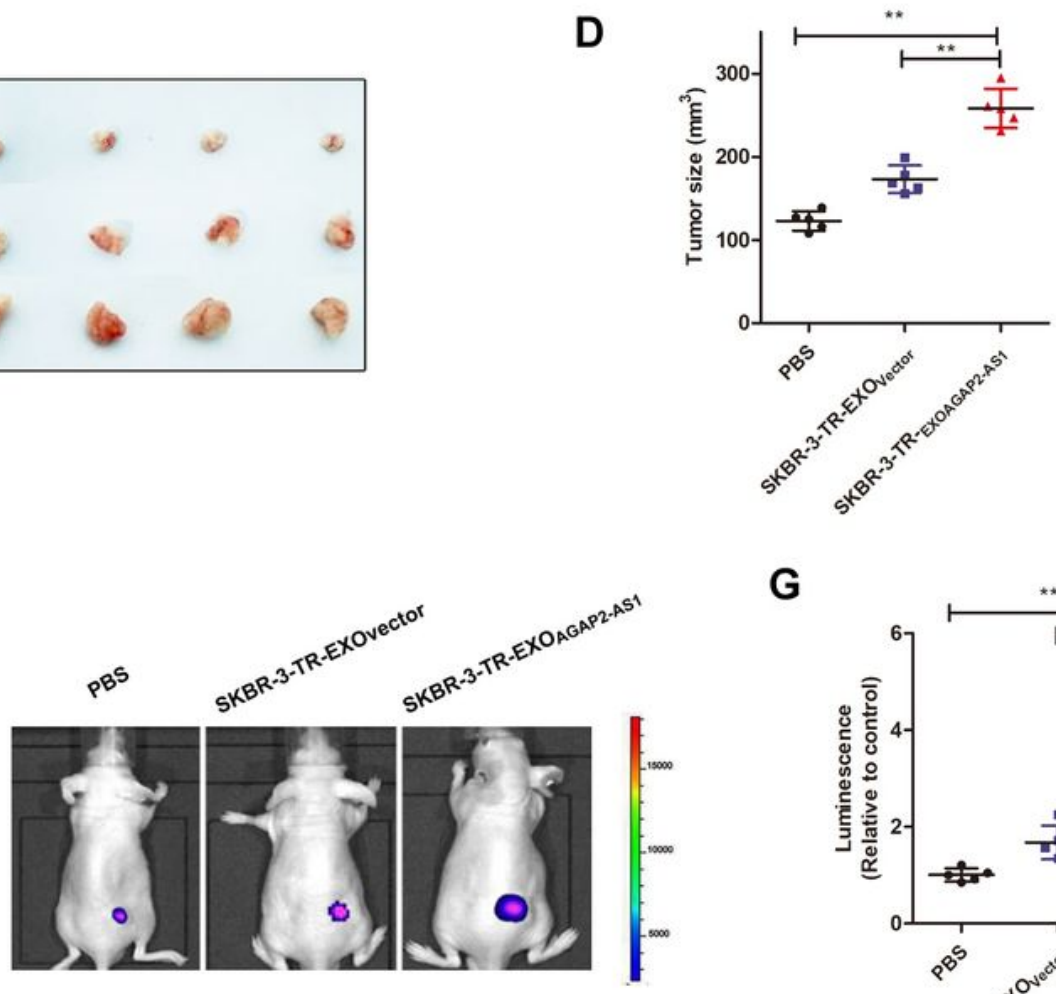

G
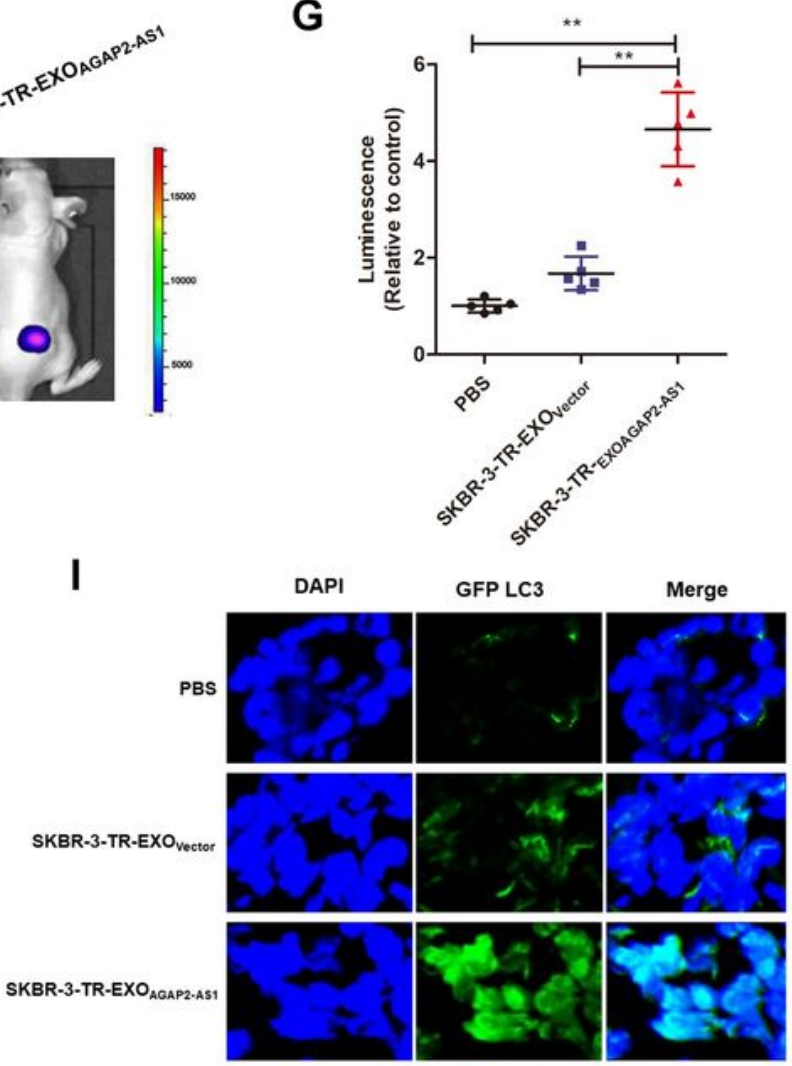

\section{Figure 5}

Exosomal AGAP2-AS1 promotes trastuzumab resistance via ATG10 in vivo (A) The schematic diagram and protocols to establish mouse xenografts. (B) The schematic diagram showed plan of trastuzumab treatment. (C) The stripped xenografts from different groups were shown. (D-E) Tumor size and tumor weight of stripped xenografts were calculated. (F-G) Dual BLI/NIRF imaging were generated and the luciferase intensity were quantified. (H) ATG10 expression was detected in established tumors from 
different groups by using IHC analysis. (I) GFP LC3 signaling was evaluated in xenografts to determine autophagy activity. ${ }^{* *} \mathrm{P}<0.01$

A

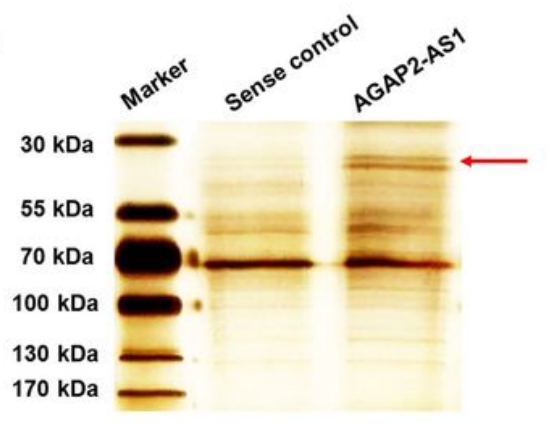

D

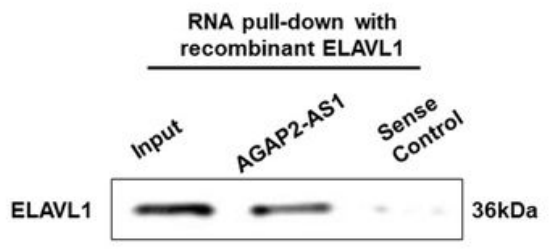

G

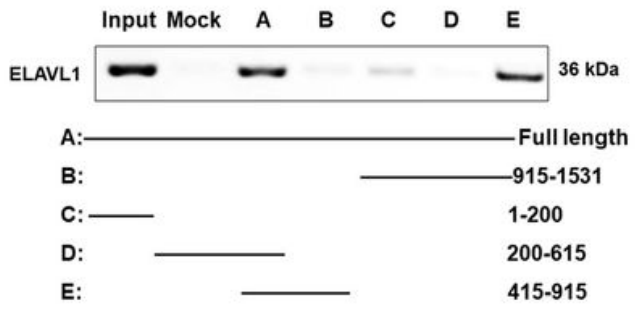

B

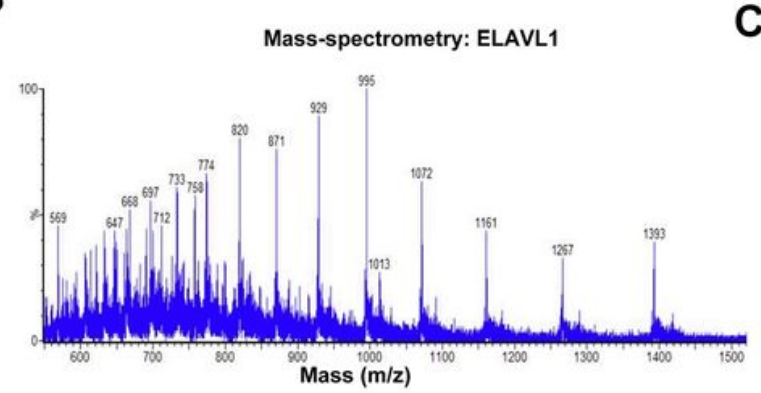

E

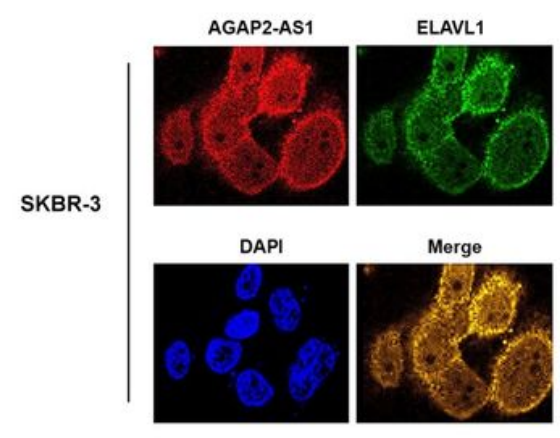

H Predicted motif sequence

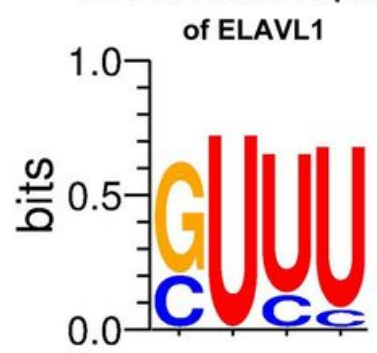

C

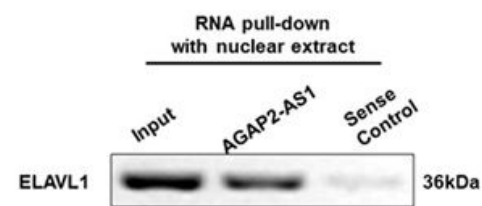

$\mathbf{F}$

- U1

- AGAP2-AS1

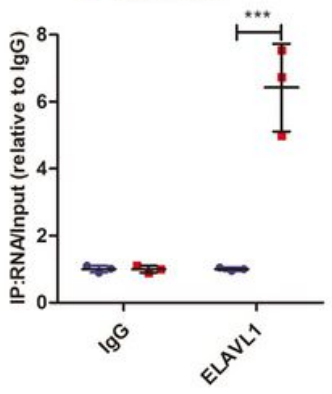

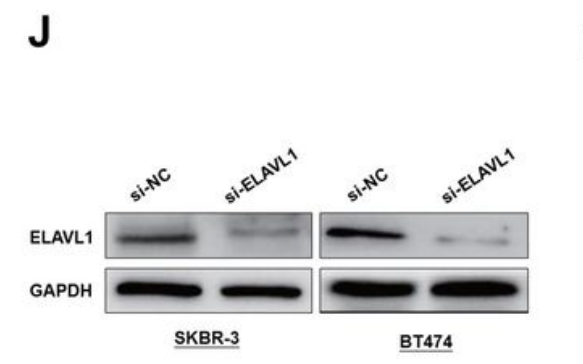

K : si-NC

L
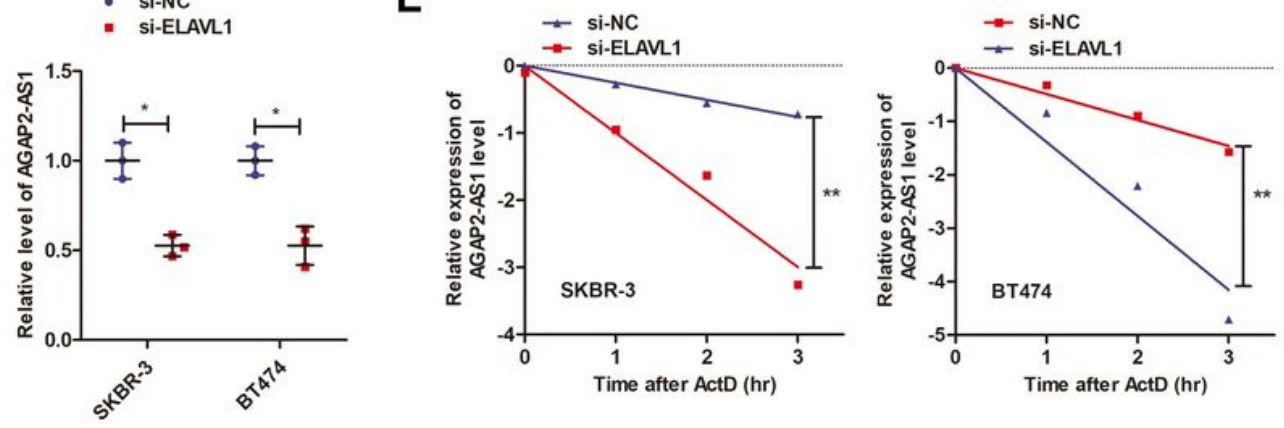

\section{Figure 6}

AGAP2-AS1 was interacted and stabilized by ELAVL1 (A) Silver-stained RNA pulldown assay usingbiotinylated AGAP2-AS1and sense control were performed, and a specific band was identified. (B) Mass spectrometry analysis of proteins from RNA pulldown, and the ELAVL1 atlas was shown. (C-D) Western blotting confirmed the interacted protein was ELAVL1. (E) RNA-FISH with specific probe verified 
that AGAP2-AS1 and ELAVL1 colocalized mostly in the cytoplasm of SKBR-3 cells. (F) RIP assay with ELAVL1 protein further confirmed the direct interaction between AGAP2-AS1 and ELAVL1. (G) Serial deletion analysis revealed that the 900-1200 nt region of AGAP2-AS1 was essential for binding with ELAVL1. (H) Predicted motif sequence of ELAVL1 was shown according to POSTAR2

(http://lulab.life.tsinghua.edu.cn/postar/index.php). (I) The 937-957 nt region of AGAP2-AS1 formed a stem-loop structure as predicted by RNAfold (http://rna.tbi.univie.ac.at//cgi-

bin/RNAWebSuite/RNAfold.cgi?). (J) ELAVL1 was silenced after transfection with specific silencing vectors. (K) qPCR showed that knockdown of ELAVL1 decreased AGAP2-AS1 expression in SKBR-3 and BT474 cells. (L) The half-life of AGAP2-AS1 RNA wasdramatically decreased in ELAVL1-silenced cells compared to that in control cells. ${ }^{*} \mathrm{P}<0.05,{ }^{*} * \mathrm{P}<0.001$

A

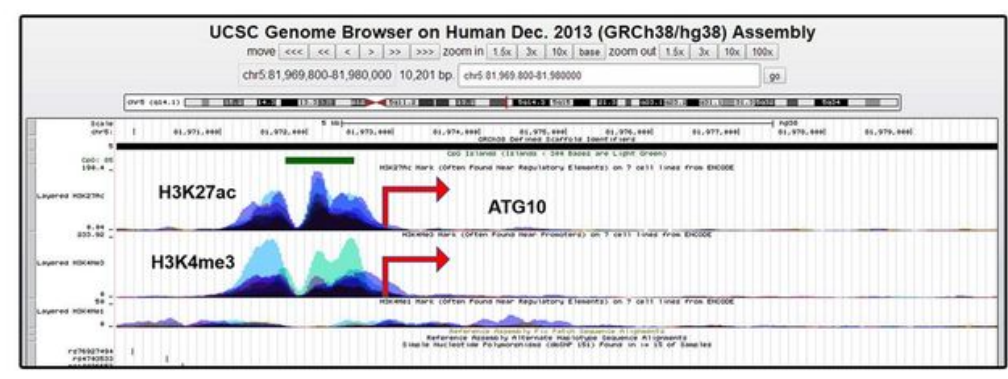

C
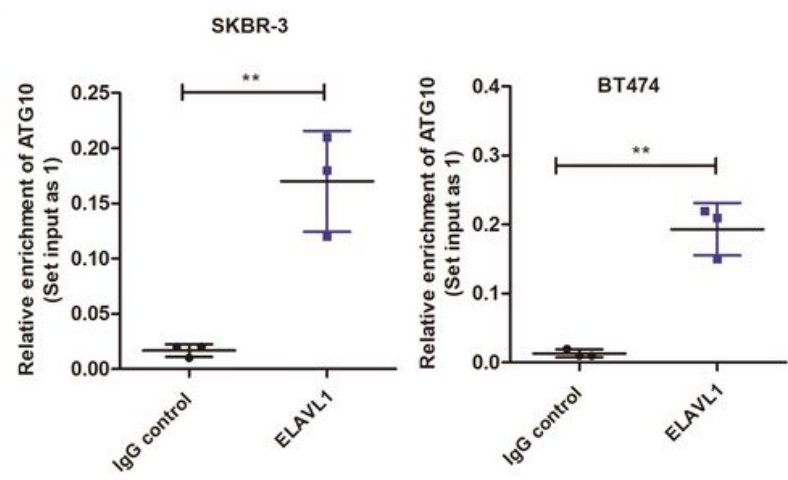

E
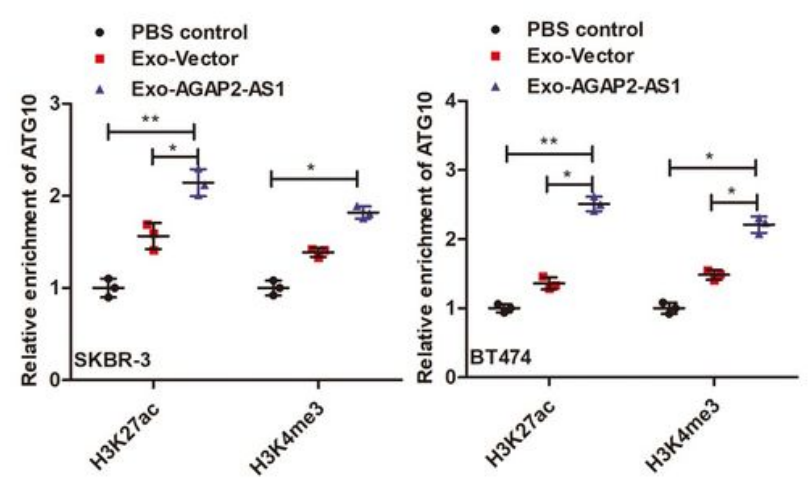

B
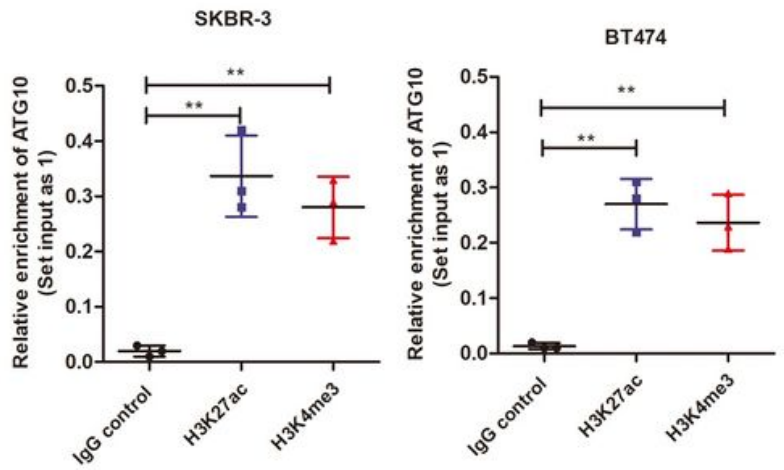

D
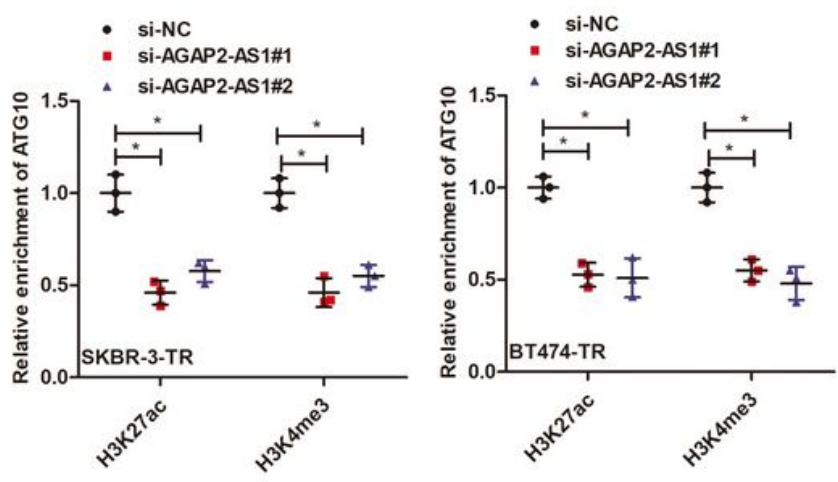

F
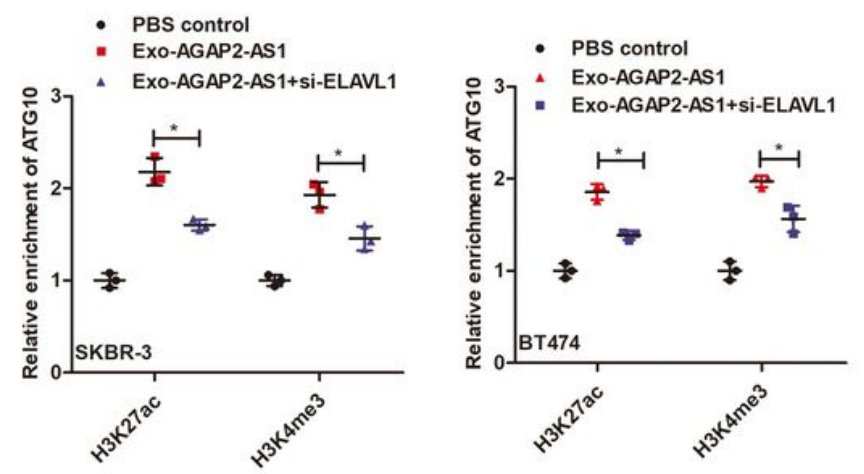

Figure 7 
AGAP2-AS1-ELAVL1 complex promote H3K4 trimethylation and H3K27 acetylation at ATG10 promoter region (A) Predictive H3K27ac and H3K4me3 binding sites were shown according to UCSC online website. (B) ChIP-qPCR showed a direct enrichment of H3K27ac and H3K4me3 at the promoter region of ATG10. (C) ChIP-qPCR verified that ELAVL1 was enriched at ATG10 promoter region. (D) knockdown of AGAP2-AS1 decreased H3K27me3 and H3K27ac enrichment in SKBR-3-TR and BT474-TR cells. (E) Enrichment of $\mathrm{H} 3 \mathrm{~K} 4 \mathrm{me} 3$ and $\mathrm{H} 3 \mathrm{~K} 27 \mathrm{ac}$ at the ATG10 promoter were increased upon treatment with exosomes secreted by AGAP2-AS1-transfected SKBR-3-TR cells. (F) Silencing ELAVL1 reversed AGAP2AS1-induced enrichment of H3K27me3 and H3K27ac. ${ }^{*} \mathrm{P}<0.05,{ }^{* * P} \mathrm{P}<0.01$

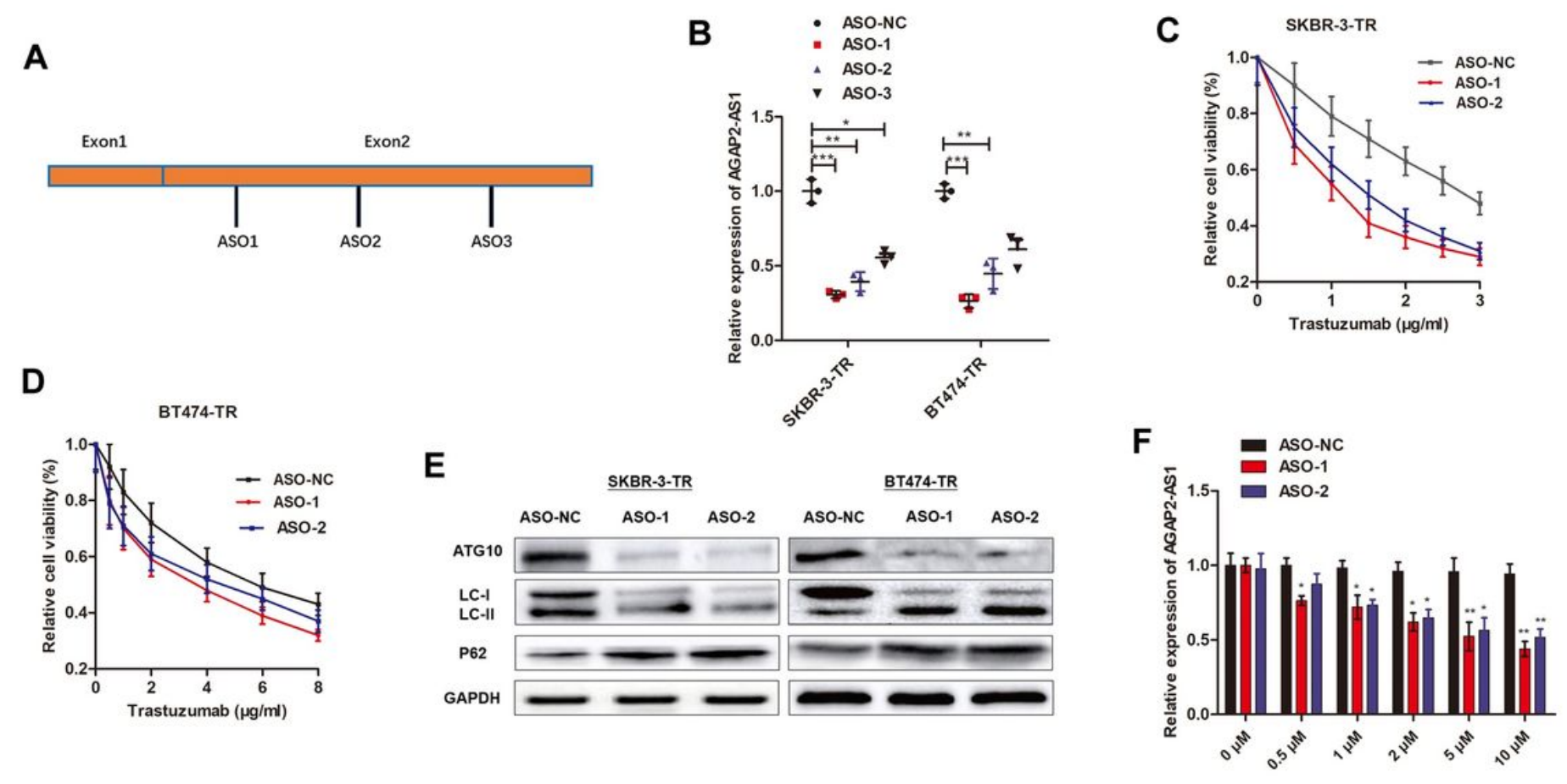

G

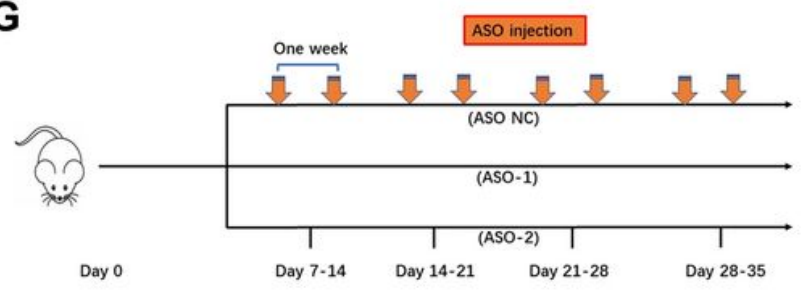

I

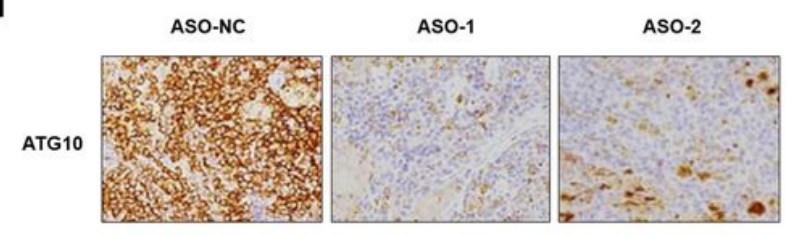

H

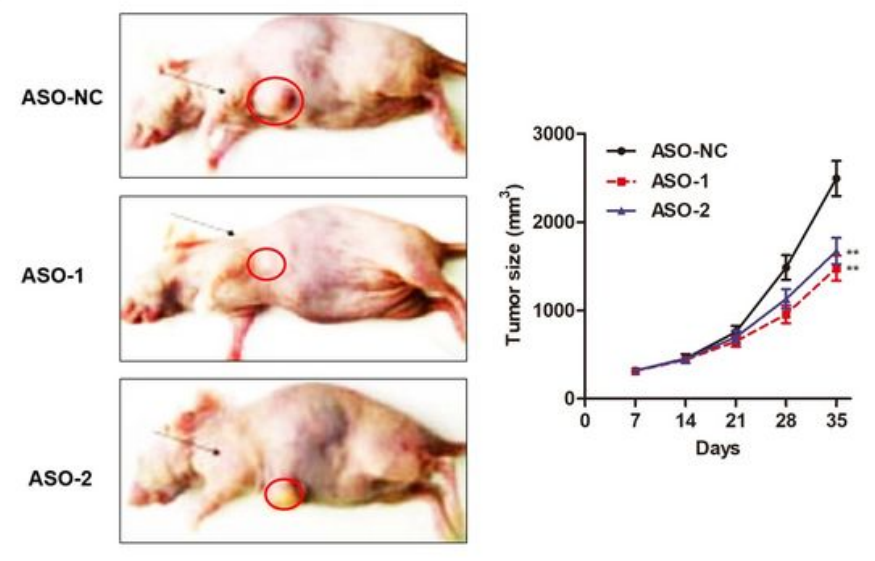

\section{Figure 8}

Potential therapeutic role of AGAP2-AS1 in BC. (A) Schematic view of ASO targets on AGAP2-AS1 gene. (B) Relative expression ofAGAP2-AS1 $48 \mathrm{~h}$ after ASO transfection were determined by qRT-PCR. (C-D) ASO treatment increases trastuzumab-induced cell cytotoxicity in SKBR-3-TR and BT474-TR cells. (E) 
Western blotting showed the suppressive effect of ASOs in autophagy activity. (F) qRT-PCR showed AGAP2-AS1 expression after ASO delivery without transfect reagents. ASO were added into SKBR-3-TR cells at theconcentration as indicated. After $48 \mathrm{~h}$, RNA was extracted, and RT-qPCR was performed. (G) Schematic view of ASO treatment xenograft model. $(\mathrm{H})$ Tumor growth was significantly suppressed in two ASO-treated groups compared to ASO-control group. (I) IHC analysis showed that ATG10 expression was suppressed in tissues treated with the two ASOs compared to control group. ${ }^{*} P<0.05,{ }^{\star *} P<0.01$, $* * * \mathrm{P}<0.001$

A

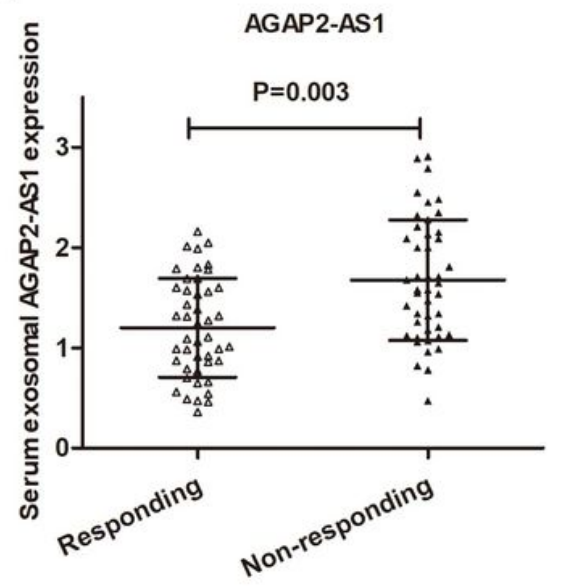

D
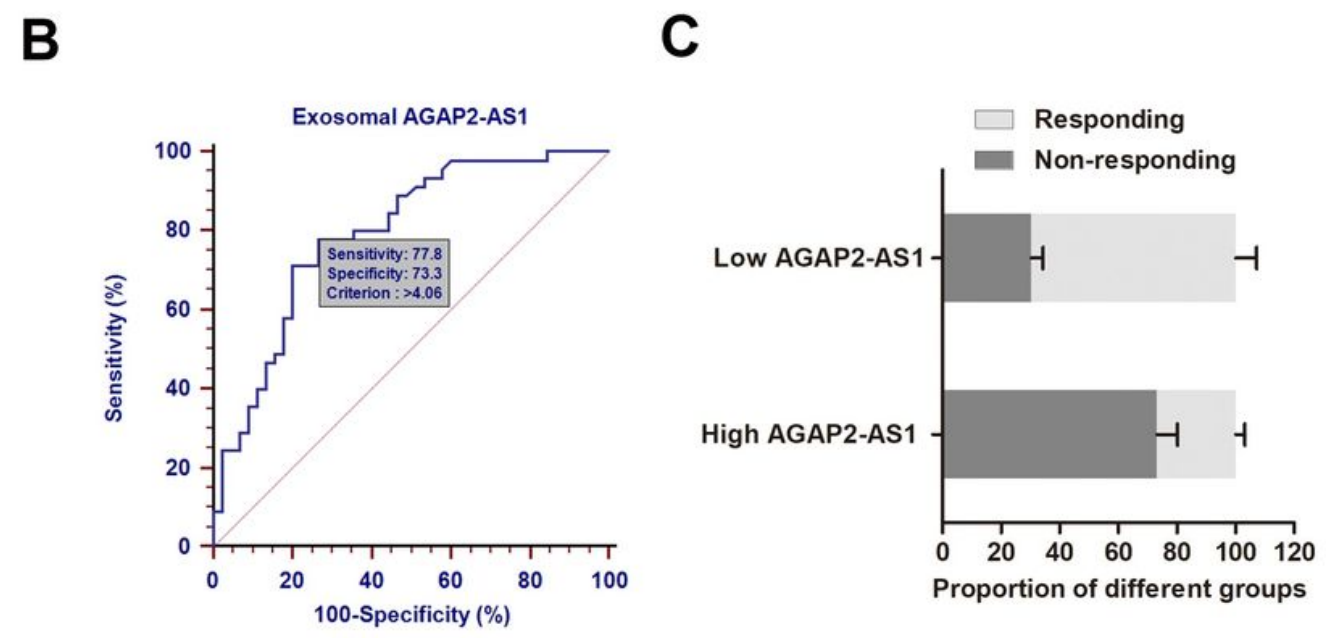

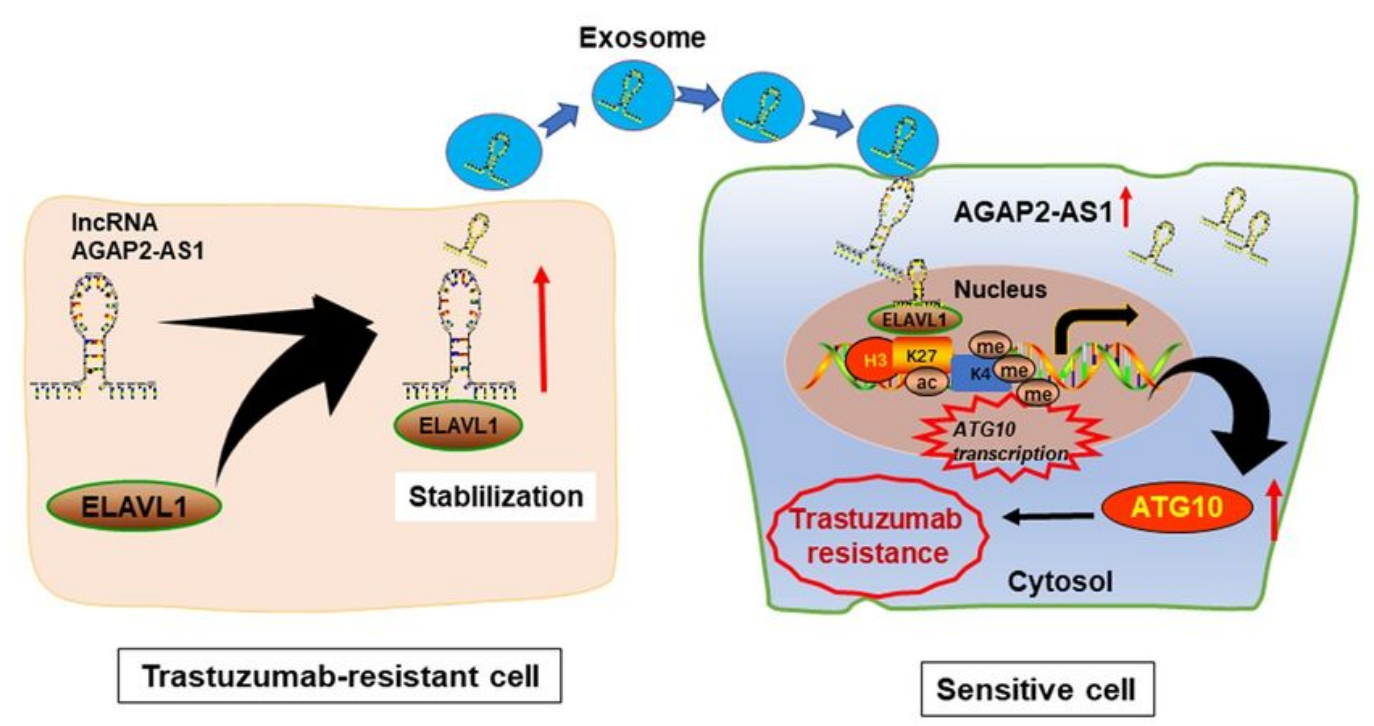

\section{Figure 9}

The clinical predictive role of circulating exosomal AGAP2-AS1 (A) Serum exosomal AGAP2-AS1 expression was detected in responding and non-responding patients. (B) ROC curve was established to evaluate the predictive role of AGAP2-AS1 in discriminating responding and non-responding patients. (C) The proportion of high or low-expressing AGAP2-AS1 patients in responding or non-responding groups were shown. (D) Schematic diagram of ELAVL1-AGAP2-AS1-ATG10 axis regulating trastuzumab resistance in $\mathrm{BC}$ via exosome. 


\section{Supplementary Files}

This is a list of supplementary files associated with this preprint. Click to download.

- Additionalfile1supplementaryTableS1.docx

- Additionalfile1supplementaryTableS1.docx 